\title{
N-Terminal Acetylation and C-Terminal Amidation of Spirulina platensis-Derived Hexapeptide: Anti-Photoaging Activity and Proteomic Analysis
}

\author{
Qiaohui Zeng ${ }^{1,2}$, Jianguo Jiang ${ }^{1}$, Jingjing Wang ${ }^{2}$, Qiuchan Zhou ${ }^{3}$ and Xuewu Zhang ${ }^{1, *}$ \\ 1 College of Food Science and Engineering, South China University of Technology, Guangzhou 510640, China; \\ z_qh2011@163.com (Q.Z.); jianguo@163.com (J.J.) \\ 2 Department of Food Science, Foshan University, Foshan 528000, China; jingjing@126.com \\ 3 Institute of Laboratory animal science, Jinan University, Guangzhou 510632, China; qiuchan@126.com \\ * Correspondence: snow_dance@sina.com; Tel./Fax: +86-2087-113-848
}

Received: 12 July 2019; Accepted: 1 September 2019; Published: 4 September 2019

\begin{abstract}
Ultraviolet (UV) irradiation is a potent inducer for skin photoaging. This paper investigated the anti-photoaging effects of the acetylated and amidated hexapeptide (AAH), originally identified from Spirulina platensis, in (Ultraviolet B) UVB-irradiated Human immortalized keratinocytes (Hacats) and mice. The results demonstrated that AAH had much lower toxicity on Hacats than the positive matrixyl ( $81.52 \%$ vs. $5.32 \%)$. Moreover, AAH reduced MDA content by $49 \%$; increased SOD, CAT, and GSH-Px activities by 103\%, 49\%, and 116\%, respectively; decreased MMP- 1 and MMP-3 expressions by $27 \%$ and $29 \%$, respectively, compared to UVB-irradiated mice. Employing isobaric tags for relative and absolute quantitation (iTRAQ)-based proteomics, 60 differential proteins were identified, and major metabolic pathways were determined. Network analysis indicated that these differential proteins were mapped into an interaction network composed of two core sub-networks. Collectively, $\mathrm{AAH}$ is protective against UVB-induced skin photoaging and has potential application in skin care cosmetics.
\end{abstract}

Keywords: photoaging; acetylation; amidation; hexapeptide; proteomics

\section{Introduction}

Solar ultraviolet (UV) light is composed of UVC (200-280 nm), UVB (280-315 nm), and UVA $(315-400 \mathrm{~nm})$. UVB can penetrate the epidermis and is particularly damaging to skin. It contributes predominantly to skin photoaging. Clinically, photoaging is characterized by coarse solar scars, roughness, dryness, wrinkles, laxity, and pigmentation [1]. UVB-induced photoaging not only damages biological macromolecules such as deoxyribonucleic acid (DNA), carbohydrates, lipids and proteins, but also decrease the activities of antioxidant enzymes in the skin such as superoxide dismutase (SOD) and glutathione peroxidase (GSH-Px) [2]. In addition, UVB-induced photoaging causes damage to the extracellular matrix (ECM) integrity in skin tissues by stimulating the production of various matrix metalloproteinases (MMP) such as MMP-1 and MMP-3 [3].

As early as the year of 2006, the micro alga Spirulina platensis extracts was revealed to possess anti-photoaging activity [4]. In addition, Neyrinck et al. showed that the oral administration of a Spirulina is able to modulate the gut microbiota and to activate the immune system in the gut, which is a mechanism that may be involved in the improvement of the hepatic inflammation in aged mice [5]. Meanwhile, numerous studies showed that Spirulina and Spirulina-derived peptides possess multiple activities such as prevention of hyperglycemia [6], antioxidant, immunomodulatory, and anti-inflammatory effects after dietary supplementation [7]. Furthermore, Souza and his research group payed much attention to the benefits of the topical application of Spirulina. Their results demonstrated 
that formulations containing Spirulina extract (at $0.1 \% w / w)$ were compatible with the skin and exerted immediate effects on the skin microrelief and hydration. Moreover, their research indicated that Spirulina nanoparticles loaded by dimethylmethoxy chromanol could significantly improve the skin pigmentation, the collagen degradation on the dermis and thereby the skin net elasticity, when they were used as supplementation to sunscreen formulation [8]. However, people have paid little attention to the anti-photoaging activity of Spirulina-derived peptides.

Recently, we identified a hexapeptide GMCCSR from trypsin hydrolysate of Spirulina platensis protein [9]. Preliminary in vitro studies showed that GMCCSR exhibited potent antioxidant and collagen-stimulating activities, and this prompts us to further explore its anti-photoaging activity in UVB-induced mice [9]. However, it is known that chemically synthesized peptides carry free amino and carboxy termini and are electrically charged in general. In order to remove this electric charge, peptide ends are often modified by N-terminal acetylation and/or C-terminal amidation. More importantly, such a modified peptide possesses some advantages: (1) mimics natural peptides due to the absence of the uncharged peptide ends, (2) resists synthetase activity by blocking the peptide ends, (3) increases cell permeability and helps binding to cell membrane, (4) enhances stability toward digestions by aminopeptidases, (5) increases bioactivity and enzyme stability by stabilizing the peptide conformation, (6) enhances activity of peptide hormones by amidation of peptides [10-13]. Hence, the purpose of this study is to investigate the effects of the acetylated and amidated hexapeptide (AAH) on anti-photoaging in UVB-irradiated Human immortalized keratinocytes (Hacats) and mice, and to understand the mechanism of action by isobaric tags for relative and absolute quantitation (iTRAQ)-based proteomics.

\section{Materials and Methods}

\subsection{Chemicals}

Trypsin was from Promega, Gaungzhou, China. PMSF (Phenylmethanesulfonyl fluoride) was from Solarbio, Guangzhou, China. MTT (3-(4,5-dimethylthiazol-2-yl)-2,5-diphenyltetrazolium bromide), thiocarbamide, DTT (dithiothreitol) and iodoacetamide were obtained from Sigma-Aldrich Co. LLC., Shanghai, China. MDA, GSH-Px, CAT and SOD assay kits were purchased from Nanjing Jiancheng Bioengineering Institute, Nanjing, China. Dulbecco's modified Eagle's medium (DMEM), Fetal bovine serum (FBS), and the antibiotic mixture (penicillin-streptomycin) were purchased from Jianyang Biotechnology Co., Ltd., Guangzhou, China. Rabbit anti-Haptoglobin, anti-Cytochrome c, anti-Nucleophosmin, anti-HSP60, anti-CA3, anti-PDHA1, anti-GAPDH were purchased from Bioworlde, Guangzhou, China. Goat anti-rabbit IgG-HRP was from Santa Cruz, CA, USA. Other reagents were of analytical grade.

\subsection{The Acetylated and Amidated Modification of Hexapeptide}

The isolation and purification of Spirulina platensis-derived hexapeptide GMCCSR were well described in previous study [9]. GMCCSR and its modification were custom synthesized by Synpeptide Co. Ltd. (Shanghai, China) using the standard Fmoc method by the use of Rink Amide. The purity of synthesized peptides was over $95 \%$.

\subsection{Cell Viability Using the MTT Method}

Human immortalized keratinocytes (Hacats) were purchased from the cell bank of CoBioer biosciences CO., LTD (Nanjing city, China). The cells were cultured in complete DMEM supplemented with $10 \%$ fetal bovine serum, $100 \mathrm{IU} / \mathrm{mL}$ penicillin, and $100 \mu \mathrm{g} / \mathrm{mL}$ streptomycin, and were incubated at $37^{\circ} \mathrm{C}$ in a damp incubator with $5 \% \mathrm{CO}_{2}$.

Subsequently, the exponentially growing cells $\left(100 \mu \mathrm{L}, 5 \times 10^{4}\right.$ cells $\left./ \mathrm{mL}\right)$ were seeded in 96-well plates for $24 \mathrm{~h}$. Removing the supernatant, cells were washed with phosphate-buffered saline (PBS) and placed in fresh PBS. UVB irradiation of cells was carried out using UV crosslinker (UVP laboratory, 
Westbury, NY, USA), five parallel tubes emitting $280-320 \mathrm{~nm}$ wavelength with a peak at $302 \mathrm{~nm}$, and cells were irradiated at the desired dosages of $35 \mathrm{~mJ} \mathrm{~m}^{-2}$, which was according our earlier publication with minor modification [9]. After UVB treatment, Hacats were incubated in complete DMEM with or without sample treatments. Following 3 days of incubation, medium was removed and 3-(4,5-Dimethylthiazol-2-yl)-2,5-diphenyltetrazolium bromide (MTT) solution (Sigma Aldrich, Los Angeles, CA, USA) was added to each well, and cells were incubated for $4 \mathrm{~h}$ at $37^{\circ} \mathrm{C}$. Negative control was MTT solution and culture media, and positive control was Matrixyl, i.e., Palmitoyl Pentapeptide-3: Palmitoyl-KTTKS. Then, $150 \mu \mathrm{L}$ dimethyl sulfoxide (DMSO) was added to each well and incubated for an additional $10 \mathrm{~min}$. The optical density was read at $490 \mathrm{~nm}$ on a SunriseTM microplate reader (TECAN, San Jose, CA, Switzerland).

\subsection{Animal Experiment}

\subsubsection{Animal}

Female Kunming (KM) mice (7-8 week) were obtained from the Animal Center of Southern Medical University (Guangzhou, China). All experimental procedures were carried out in accordance with the standard guidelines for the care of animals that were approved by Jinan University Committee for Animal Care and Use. Mice were housed at a temperature of $23 \pm 2{ }^{\circ} \mathrm{C}$ and humidity of $55 \% \pm 10 \%$ specific pathogen-free environment with a $12 \mathrm{~h}$ light/dark cycle and given free access to standard laboratory diet and water. Prior to the start of the experiment, mice were acclimatized for at least 7 days.

\subsubsection{UV Irradiation and Peptide Treatment}

UVB has been thought to be responsible for the damaging effects in the skin. Therefore, we used a UVB lamp apparatus (peak at $302 \mathrm{~nm}$ ) for construction of a photoaged skin animal model in this study. The dorsal skin of mice was exposed to UVB light for 10 weeks of three times per week at an intensity of $60 \mathrm{~mJ} / \mathrm{m}^{2}$, which was closed to four minimally erythemogenic doses (MED). During the experiment, once erythema, blisters, and erosion occurred in the dorsal skin of mice, the irradiation was stopped for 2-3 days, which could continue until the symptoms disappeared.

We randomly divided $32 \mathrm{KM}$ mice into four groups of eight mice: no UVB exposure (normal), UVB irradiation (model), UVB + Matrixyl (positive control Matrixyl, i.e., Palmitoyl Pentapeptide-3: Palmitoyl-KTTKS), and UVB + AAH groups (experimental group, acetylated and amidated hexapeptide (AAH)).

Hair in dorsal skin of mice was removed within an area of $2.5 \times 3 \mathrm{~cm}^{2}$ using a lady shaver, and the mice were acclimatized for two days before the experiment. A total of $100 \mu \mathrm{L}$ of AAH $(10 \mathrm{mg} / \mathrm{mL})$, Matrixyl (10 mg/mL), and vehicle (no UVB exposure group and UVB irradiation group) were applied on the shaved dorsal skin. In this study, we used a solvent mixture of ethanol:water:propylene glycol $=3: 3: 4$ (v:v:v) as a vehicle. This solvent mixture is the proper vehicle to fully solve the peptide. We topically applied all samples (100 $\mu \mathrm{L} /$ each mouse) on the dorsal skin of mice, and then gave enough time to be absorbed into the skin (about $2 \mathrm{~h}$ ). The skin care product Matrixyl was used as the positive control. The mice were exposed to UVB radiation three times per week at $60 \mathrm{~mJ} / \mathrm{cm}^{2}$ for 10 weeks. We euthanized the mice in a humane way at the end of the study. Mice were anesthetized with ether and then executed cervical dislocation without pain. Then, skin specimens from the central dorsum of the mice were obtained.

\subsubsection{Histological Examination and Moisture Content Test of Skin}

The dorsal skin of mice was photographed under anesthesia by diethyl ether inhalation at the end of the study. An area of $1.0 \times 1.0 \mathrm{~cm}^{2}$ was harvested freshly and fixed in $10 \%$ neutral buffered formalin. The degree of skin structure alteration and elastosis were assessed microscopically using Haematoxylin-eosin (H\&E) staining. To quantify epidermal thickness following UV exposure, 
measurement was conducted at 10 randomly selected locations per slide using an optical microscope with 200× magnification. Histological alterations were evaluated and quantified through the image analysis program Image Pro Plus 6.0 (Silver Spring, MD, USA).

Skin $(0.2 \mathrm{~g})$ was quickly cut and precisely weighted $\left(\mathrm{w}_{1}\right)$, then it was moved into the oven, and dried at $80^{\circ} \mathrm{C}$ to constant weight $\left(\mathrm{w}_{2}\right)$. Therefore, the skin moisture percentage could be determined by the following formula: the skin moisture percentage $(\%)=\left(w_{1}-w_{2}\right) / w_{1} \times 100$.

\subsubsection{Determination of SOD, CAT, GSH-Px, and MDA in Skin Tissue}

The harvested skin tissue $(0.4 \mathrm{~g})$ was homogenized $(10,000 \mathrm{rpm}, 20 \mathrm{~s})$ in nine volumes of $0.9 \%$ saline $\left(4{ }^{\circ} \mathrm{C}\right)$ to obtain the $10 \%$ skin tissue homogenate. The total supernatant was used for protein content, SOD, CAT, GSH-Px and MDA determination according to the manufacturer's protocols.

\subsubsection{Determination of MMP-1 and MMP-3 in Skin Tissue}

Skin tissue $(0.4 \mathrm{~g})$ was homogenized $(10,000 \mathrm{rpm}, 20 \mathrm{~s})$ in nine volumes of PBS $\left(4{ }^{\circ} \mathrm{C}\right)$ to obtain the $10 \%$ skin tissue homogenate. The undissolved pellet was removed by centrifugation at $3000 \times g$ for $20 \mathrm{~min}$ at $4{ }^{\circ} \mathrm{C}$, and the total supernatant was saved for the subsequent assays. Secreted MMP- 1 and MMP-3 were estimated using ELISA kits (Thermo scientific, Waltham, MA, USA) and protein content was determined according to the manufacturer's instructions.

\subsection{Proteomics}

\subsubsection{Sample Preparation and Labeling}

A total of four samples were collected as the target tissue for iTRAQ analysis at Shanghai Majorbio BioMedical Technology Co., Ltd. The samples were lysed to enrich the phosphorylated proteins with phosphoprotein enrichment kit according to the manufacturer's instructions. The protein concentration was determined by the Bradford method. Afterwards, $150 \mu \mathrm{g}$ of protein for each sample was mixed with Dithitol (DTT, final concentration of $10 \mathrm{mM}$ ), reacted for $30 \mathrm{~min}$ at $56^{\circ} \mathrm{C}$, then, Iodoacetamide (IAA, final concentration of $20 \mathrm{mM}$ ) was added keeping in darkness for $30 \mathrm{~min}$. Then, five volumes of cold acetone were precipitated for $2 \mathrm{~h}$ at $-20^{\circ} \mathrm{C}$ and then centrifuged at $12,000 \mathrm{rpm}$ for $20 \mathrm{~min}$ at $4{ }^{\circ} \mathrm{C}$. The precipitation was dissolved by $20 \mu \mathrm{L}$ TEAB buffer with $1 \mathrm{M}$ urea. Next, Trypsin (1/50 protein) was added and reacted for $15 \mathrm{~h}$ at $37^{\circ} \mathrm{C}$. Trichloroacetic acid (TFA, final concentration of $0.5 \%$ ) was added to stop the hydrolysis reaction. The deposit was collected and dried by vacuum freeze dryer. Then, the sample $(100 \mu \mathrm{g})$ was dissolved in dissolution buffer of the iTRAQ kit and reacted with $40 \mu \mathrm{L}$ reducing reagent and $70 \mu \mathrm{L}$ Isopropyl alcohol, and then $40 \mu \mathrm{L}$ Milli-Q water was added after $2 \mathrm{~h}$ incubation. All the labeled samples from different treatment groups were pooled and freeze-dried.

\subsubsection{Sample Fractionation and LC-MS/MS Analysis}

Dried peptides were resuspended with buffer A: water (formic acid and ammonia water was used to adjust the $\mathrm{pH}$ to 10). Buffer B was $100 \%$ acetonitrile (ACN). Detection wavelengths were set as 214 and $280 \mathrm{~nm}$, meanwhile, flow rate was $200 \mu \mathrm{L} / \mathrm{min}$. The $60 \mathrm{~min}$ gradient comprised of $0-5 \%$ buffer B for $5 \mathrm{~min}, 5-25 \%$ buffer B for $35 \mathrm{~min}, 25-80 \%$ buffer B for $5 \mathrm{~min}, 80 \%$ buffer B for $5 \mathrm{~min}, 80-100 \%$ buffer B for $1 \mathrm{~min}$, and finally $100 \%$ buffer B for $9 \mathrm{~min}$. The combined samples were separated into 20 fractions.

Subsequently, the collected fractions were pooled according to the chromatogram profile based on the peak intensity and the products dried in a vacuum for LC-MS/MS analysis.

\subsubsection{Data Analysis}

The MS/MS spectra were extracted and searched against the database with Uniform Resource Locator (URL) of http://www.uniprot.org/proteomes/UP000000589 using Mascot 2.3.02 software (Boston, MA, USA). Search parameters were set as follows: (1) type of search: MS/MS Ion search; 
(2) Enzyme: trypsin; (3) Fragment Mass Tolerance: \pm 0.05 Da; (4) Mass Value: Monoisotopic; (5) Variable modifications: Gln- > pyro-Glu (N-term Q), Oxidation (M), iTRAQ8plex (Y); (6) Peptide Mass Toletance: 10 ppm; (7) Instrument type: Default; (8) Max Missed Cleavages: 1; (9) Fixed modification: Carbamidomethyl (C), iTRAQ8plex (N-term), iTRAQ8plex; (10) Protein Mass: Unrestricted.

The cellular component, molecular function, and biological process were annotated by GO (Gene Ontology) database (http://www.geneontology.org/). The signaling pathways of proteins were elucidated by searching against the Kyoto Encyclopedia of Genes and Genomes database (http://www.genome.jp/kegg/pathway.html). The protein-protein interaction network was analyzed by Search Tool for the Retrieval of Interacting Genes/Proteins (STRING) software (http://string.embl.de/).

\subsubsection{Western Blot}

The extraction of total protein was carried out according to the extraction method in the experiment of iTRAQ. The quantification of the protein was carried out in strict accordance with the BCA kits. Subsequently, $30 \mu \mathrm{g}$ of the sample was taken slowly from the micro-injector and added to the sample wells, meanwhile, $5 \mu \mathrm{L}$ of protein markers were loaded and followed by electrophoresis, transfer, blocking, primary incubation, secondary incubation, and dyeing process. Among them, slightly different conditions were used in the transfer process, the conditions for most antibodies were $300 \mathrm{~mA}$ and $40 \mathrm{~min}$, but $300 \mathrm{~mA}$ and $60 \mathrm{~min}$ for HSP60, $300 \mathrm{~mA}$ and $20 \mathrm{~min}$ for Cytochrome c. All antibody concentrations during the incubation were 1:1000 dilution. After the dyeing wass finished, pictures were taken or scanned, recording the analysis results.

\subsection{Statistical Analysis}

All assays were carried out in triplicate, and the experimental results were expressed as means \pm standard deviations. Statistical analysis was performed by SPSS 16 (SPSS Inc., Chicago, IL, USA). Data were analyzed using the least significant difference (LSD) method by analysis of variance, and the value differences were considered to be significant when $p<0.05$. The spectrum data from iTRAQ results were submitted for protein identification, and a database search was carried out using ProteinPilot Software 4.5 (AB SCIEX, Seattle, WA, USA) to perform database searches. The database used was the SwissProt_2013_09 (total sequence 540958). The search parameters used were as follows: Cysteine alkylation with MMTS; Trypsin Digestion; Triple TOF 5600; ID focus with Biological modifications; Search effort with thorough ID. A decoy database search strategy was used to determine the false discovery rate (FDR) for protein identification. The criteria for protein identification was set to FDR $<0.1 \%$.

\section{Results}

\subsection{The Toxicity on Hacats and the Protective Effect on the Damaged Hacats}

By MTT assay, the surviving rates of Hacats treated by Spirulina platensis-derived hexapeptide GMCCSR, AAH and matrixyl were $99.18 \% \pm 6.97 \%, 81.52 \% \pm 6.26 \%$, and $5.32 \% \pm 1.83 \%$, respectively. Then, the Hacats were irradiated by UVB, and the damaged Hacats were treated with the peptides again. The surviving rates corresponding to Spirulina platensis-derived hexapeptide GMCCSR, AAH and matrixyl treatment were $112.97 \% \pm 10.73 \%, 137.14 \% \pm 8.42 \%$, and $121.56 \% \pm 1.42 \%$, respectively, and the UVB control was set as $100 \%$. Comparing with the unmodified hexapeptide, the surviving rates of Hacats treated by AAH reduced from $99.18 \% \pm 6.97 \%$ to $81.52 \% \pm 6.26 \%$, which was still above $80 \%$. Thus, the follow-up step was to conduct further experiments to explore its protection effects for the UVB-damaged Hacats. In addition, the modified hexapeptide exhibited significantly lower toxicity $(p<0.05)$ and significantly stronger protective effect $(p<0.05)$ on Hacats than the positive control matrixyl. 


\subsection{Morphology, Thickness, and Moisture Content of the Dorsal Skin on Mice}

The morphological observation indicated that the dorsal skin of UVB-irradiated mice had distinct features: dark-brown color, deep wrinkle, relaxable surface, and local aging and necrosis appeared on the surface cortex (Figure 1B). After matrixyl treatment, the appearance of skin was improved, but was still a little bit brown, with clear signs of UVB irradiation (Figure 1C). However, the dorsal skin of mice treated with AAH (Figure 1D) was greatly improved, which was better than the positive control matrixyl group, and was most similar to normal group (Figure 1A): smooth surface, rosy color, satiation, no signs of relaxation and wrinkle. Notably, Figure 1(D1) also showed irregular epidermal thickening, and it is possible that there still exists some inflammation. Anyway, the extent of thickening was much smaller than those of the model group (Figure 1(B1)) and positive drug group (Figure 1(C1)), although it was slightly greater than that of the normal group (Figure 1(A1)). Furthermore, the histological examination by haematoxylin-eosin (H\&E) staining displayed that the mice skin in the normal group had thin cortex, regularly arranged collagen fibrils, plump subcutaneous hair follicles and sebaceous glands (Figure 1A). The skin in the UVB-irradiation group had irregularly thickened cortex, broken nuclei, vacuolar degeneration of epidermal basal cells, and inflammatory cells (lymphocytes and monocytes) infiltration of the dermis (Figure 1(B1)). In the positive control matrixyl group, although the cortex became thinner, there was still slight vacuolar degeneration and inflammatory cells infiltration (Figure 1(C1)). While in the AAH treatment group, the cortex was further thinned, and followed by disappearance of vacuolar degeneration and inflammatory cells infiltration (Figure 1(D1)). In a word, these results suggest that AAH possessed better effects in improving mice skin than the positive control matrixyl.
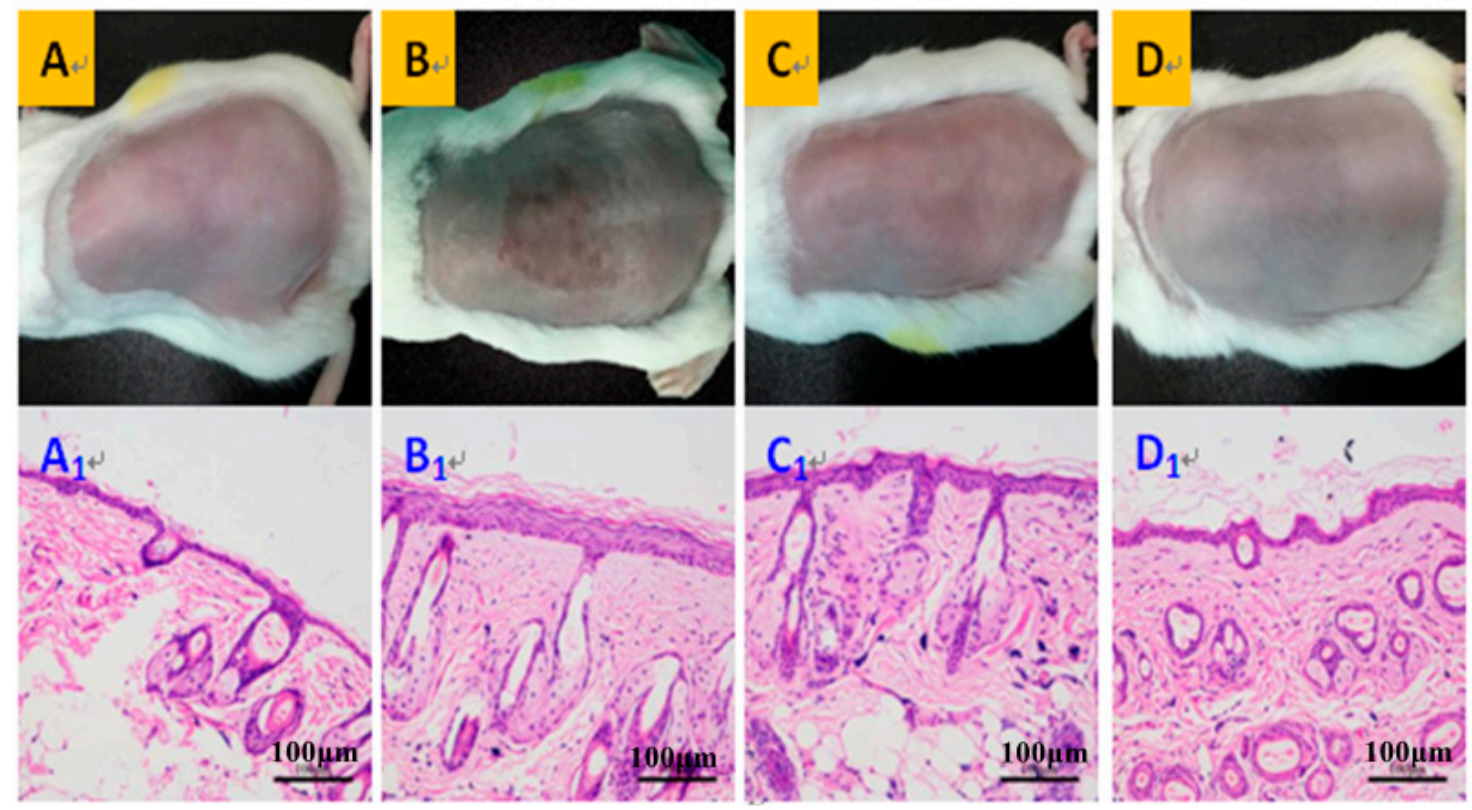

Figure 1. Morphological features (A-D) and histological examination (A1-D1, 200X) of the dorsal skin of mice. Normal control (A,A1), UVB irradiation group (B,B1), positive control matrixyl group (C,C1), and the acetylated and amidated hexapeptide group (D,D1).

By measuring the thickness of the dorsal skin (Figure 2), the results showed that UVB irradiation significantly $(p<0.05)$ increased the thickness of skin from $13.1 \mu \mathrm{m}$ in the normal group to $40.2 \mu \mathrm{m}$; the positive control matrixyl decreased $40.2 \mu \mathrm{m}$ of thickness in the UVB irradiation group to $23.8 \mu \mathrm{m}$, but still significantly $(p<0.05)$ greater than $13.1 \mu \mathrm{m}$ in the normal group; and the thickness of skin in AAH group $(13.5 \mu \mathrm{m})$ was close to $13.1 \mu \mathrm{m}$ in the normal group. Further evaluation of moisture content indicated that UVB irradiation significantly $(p<0.05)$ decreased the moisture content $(58 \%)$, compared to normal group (63.8\%); the positive control matrixyl increased the moisture content $(62.4 \%)$, 
especially, AAH significantly $(p<0.05$ ) elevated the moisture content to $63.2 \%$, compared to UVB irradiation group. This suggests that $\mathrm{AAH}$ was also better than the positive control matrixyl in terms of skin thickness and moisture content.

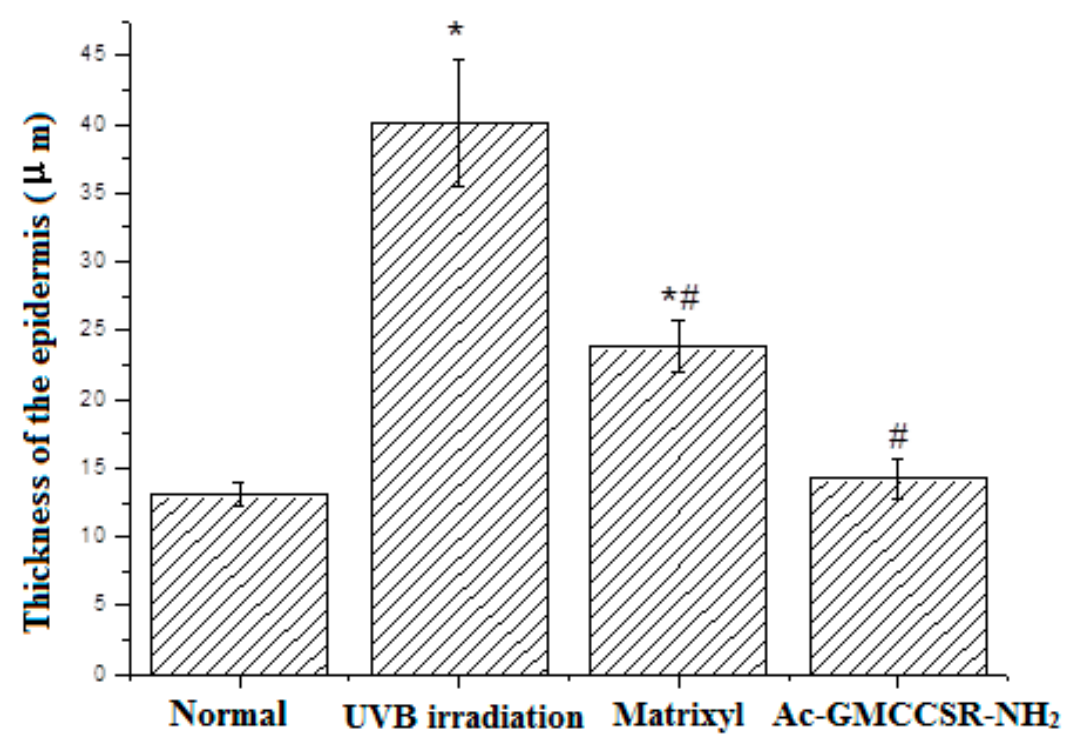

(A)

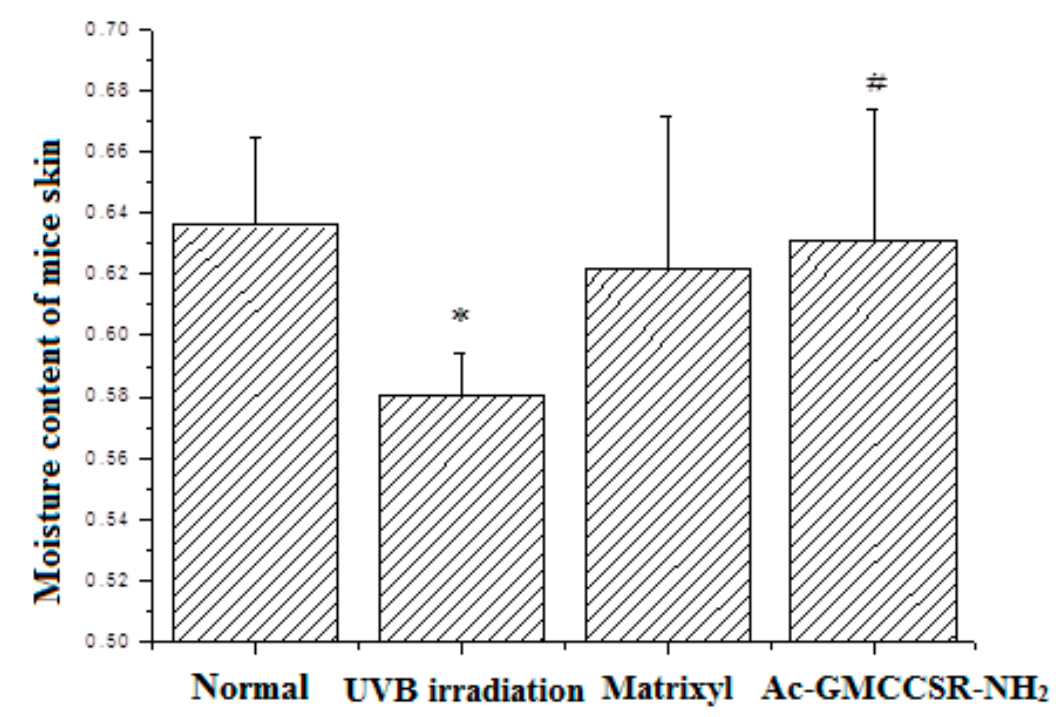

(B)

Figure 2. Thickness (A) and moisture content (B) of the dorsal skin of mice. Normal control, UVB irradiation group, positive control matrixyl group, the acetylated and amidated hexapeptide group (Ac-GMCCSR-NH $\mathrm{N}_{2}$ ). ${ }^{*}$ significant compared to normal group, \# significant compared to UVB irradiation group.

\subsection{Effects on MDA, SOD, CAT, GSH-Px, MMP-1, and MMP-3 in Skin Tissue of Mice}

As shown in Figure 3A, UVB irradiation caused the significant $(p<0.05)$ increase of MDA from $5.8 \mathrm{nmol} / \mathrm{mg}$ in the normal mice to $9.8 \mathrm{nmol} / \mathrm{mg}$ in UVB-irradiated mice. Matrixyl treatment decreased the increased MDA from 9.8 to $7.9 \mathrm{nmol} / \mathrm{mg}$, while AAH significantly $(p<0.05)$ decreased the increase from 9.8 to $4.0 \mathrm{nmol} / \mathrm{mg}$, up to $49 \%$, even lower than $5.8 \mathrm{nmol} / \mathrm{mg}$ in the normal group.

For antioxidant enzymes SOD, CAT, and GSH-Px, there was an identical trend in their alterations: UVB irradiation decreased the activities of SOD, CAT, and GSH-Px, but they were increased in the group of matrixyl treatment and in the group of treatment with AAH. Specifically, the activities 
of SOD in normal, UVB-irradiation, matrixyl, and AAH treatment groups were 57.5, 19.1, 4.7, and $38.8 \mathrm{U} / \mathrm{mg}$, respectively (Figure 3B); the activities of CAT in normal, UVB-irradiation, matrixyl, and AAH treatment groups were 13.4, 7.1, 8.2, and 10.6 U/mg, respectively (Figure 3C); the activities of GSH-Px in normal, UVB-irradiation, matrixyl, and AAH treatment groups were 130.3, 48.8, 105.1, and 105.2 U/mg, respectively (Figure 3D).

Moreover, Figure 3E shows that the expression of MMP-1 in UVB irradiation group $(150.2 \mathrm{ng} / \mathrm{mg})$ was increased compared to normal group $(90 \mathrm{ng} / \mathrm{mg}$ ), but such an increase was slowed down in the matrixyl treatment group $(120.1 \mathrm{ng} / \mathrm{mg})$ and the modified hexapeptide treatment group $(109.8 \mathrm{ng} / \mathrm{mg})$. Similarly, Figure 3F displayed that the expression of MMP-3 in the UVB irradiation group $(82.6 \mathrm{ng} / \mathrm{mg})$ was also increased compared to normal group $(71.2 \mathrm{ng} / \mathrm{mg})$, while both matrixyl and the modified hexapeptide decreased the expression of MMP-3 to 63.4 and $58.8 \mathrm{ng} / \mathrm{mg}$, respectively.

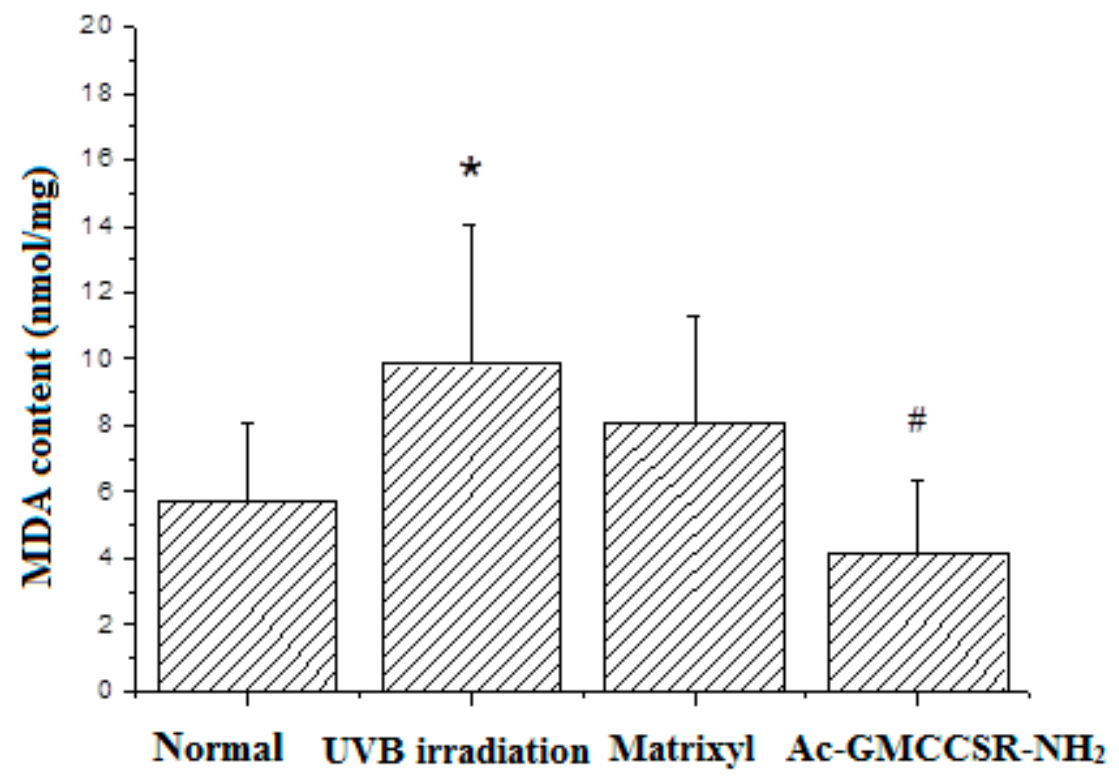

(A)

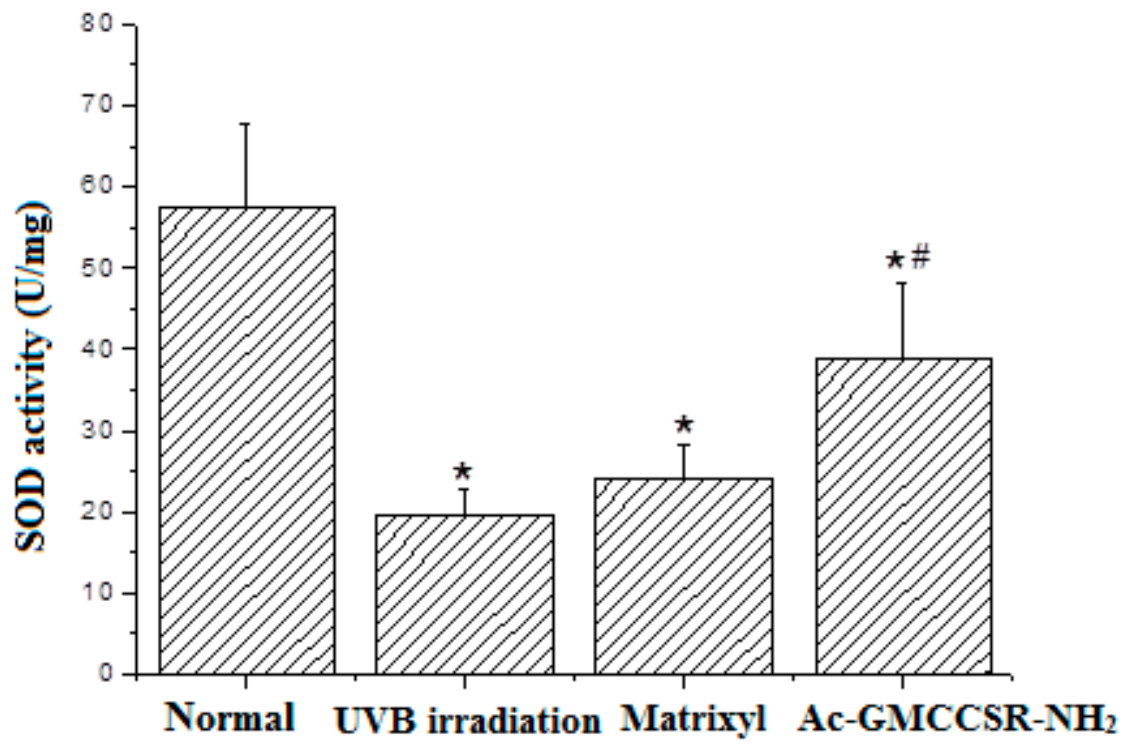

(B)

Figure 3. Cont. 


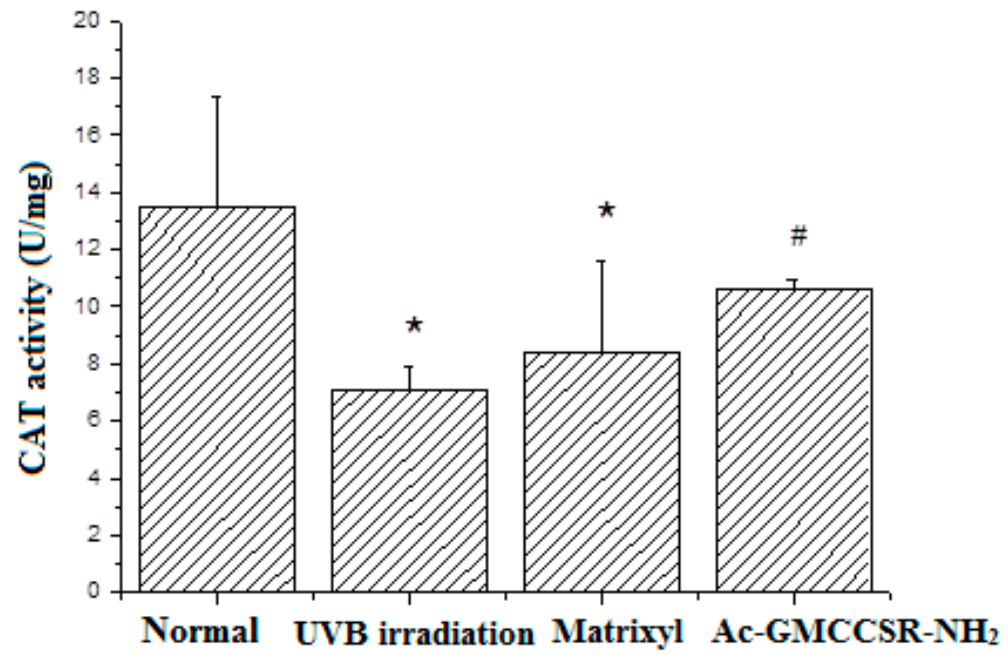

(C)

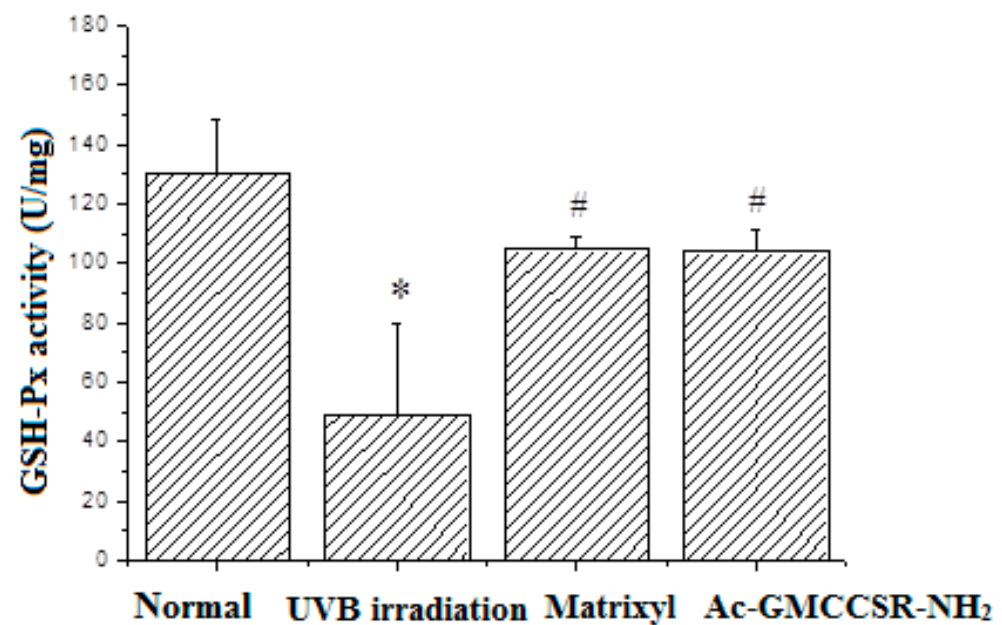

(D)

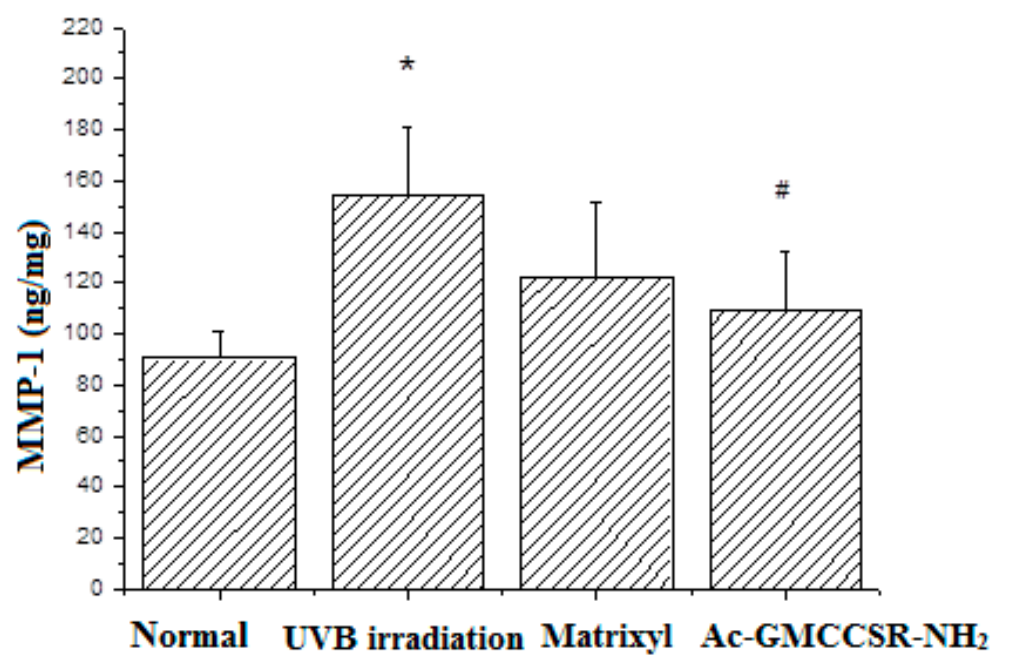

(E)

Figure 3. Cont. 


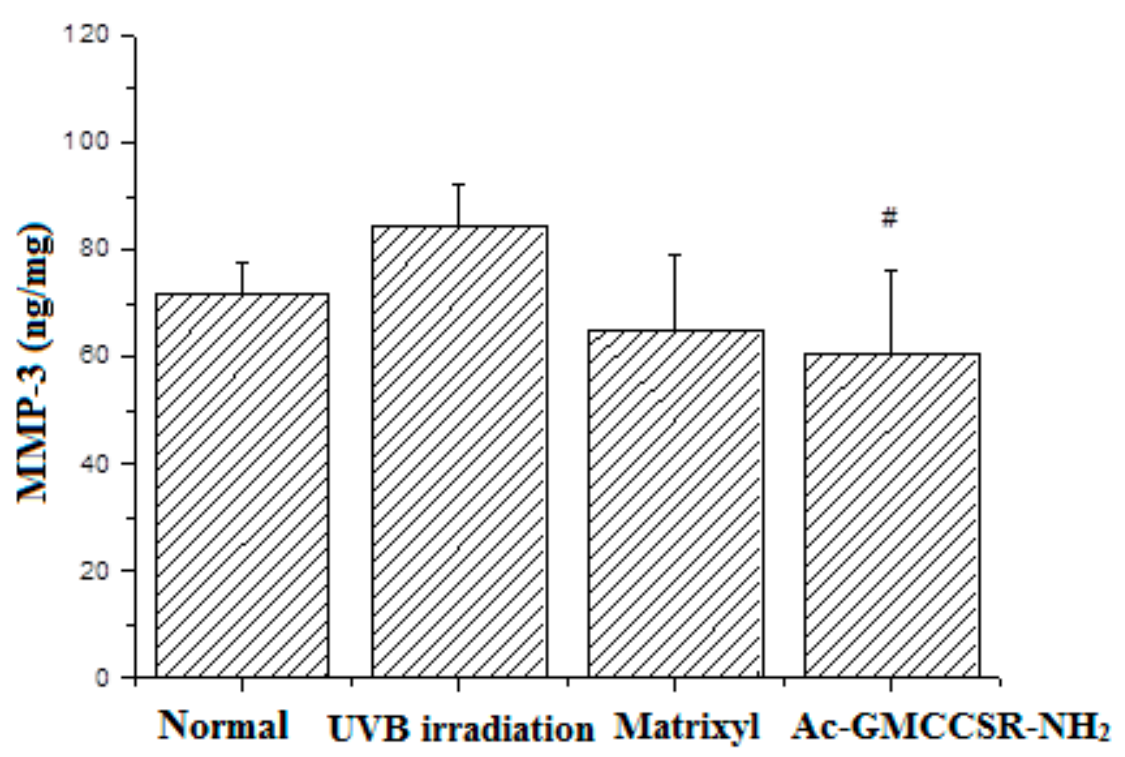

(F)

Figure 3. Effects on MDA (A), SOD (B), CAT (C), GSH-Px (D), MMP-1 (E), and MMP-3 (F) in skin tissue of mice. Normal control, UVB irradiation group, positive control matrixyl group, and the acetylated and amidated hexapeptide group (Ac-GMCCSR-NH $\mathrm{H}_{2}$ ). ${ }^{*}$ significant compared to normal group, \# significant compared to UVB irradiation group.

\subsection{Proteomic Analysis of Skin Tissue in Mice}

In order to explore molecular mechanism of protective effects on mice skin exerted by AAH, the iTRAQ-based proteomics was performed on the skin tissue from mice treated with UVB irradiation (model group) and with UVB + AAH (experimental group). The results showed that 60 differential proteins were identified, 33 proteins were up-regulated, and 27 proteins were down-regulated proteins (Table 1). Among them, significantly up-regulated proteins include: PYGM_MOUSE (1.9 fold), ODPA_MOUSE (1.6 fold), AT2A1_MOUSE (1.6 fold), TITIN_MOUSE (1.9 fold), ECP3_MOUSE (1.8 fold); significantly down-regulated proteins include: RS21_MOUSE ( -1.6 fold), K1C17_MOUSE ( -1.8 fold), HPT_MOUSE ( -3.4 fold), K1C28_MOUSE ( -1.8 fold), K1C27_MOUSE ( -1.7 fold), K1C16_MOUSE ( -1.6 fold), K2C71_MOUSE (-2.1 fold), K2C6A_MOUSE ( -3.2 fold $)$.

Table 1. Differential proteins related to the protection effect of the acetylated and amidated hexapeptide.

\begin{tabular}{|c|c|c|c|c|c|c|c|}
\hline Accession & B & D & FC (D/B) & $\log 2 \mathrm{FC}(\mathrm{D} / \mathrm{B})$ & $p$-Value & Significant & Regulate \\
\hline CO6A1_MOUSE & 0.827951 & 0.345235 & 0.416976 & -1.26197 & 0.002326 & yes & down \\
\hline RS21_MOUSE & 2.443534 & 0.794362 & 0.325087 & -1.6211 & 0.003971 & yes & down \\
\hline GPDM_MOUSE & 0.82043 & 1.132509 & 1.380384 & 0.46507 & 0.00559 & yes & up \\
\hline RS3A_MOUSE & 1.185781 & 0.698351 & 0.588937 & -0.76381 & 0.00586 & yes & down \\
\hline K1C17_MOUSE & 1.31876 & 0.385973 & 0.292679 & -1.77261 & 0.006467 & yes & down \\
\hline HPT_MOUSE & 3.841142 & 0.372877 & 0.097075 & -3.36476 & 0.006876 & yes & down \\
\hline NF1_MOUSE & 0.887495 & 1.288372 & 1.451696 & 0.537739 & 0.007991 & yes & up \\
\hline ENOB_MOUSE & 0.373393 & 1.004882 & 2.69122 & 1.42826 & 0.008388 & yes & up \\
\hline A2M_MOUSE & 1.374173 & 1.076648 & 0.783488 & -0.35202 & 0.008462 & yes & down \\
\hline CAH3_MOUSE & 0.402064 & 0.995416 & 2.475768 & 1.307876 & 0.008729 & yes & up \\
\hline
\end{tabular}


Table 1. Cont.

\begin{tabular}{|c|c|c|c|c|c|c|c|}
\hline Accession & B & D & FC (D/B) & $\log 2 \mathrm{FC}(\mathrm{D} / \mathrm{B})$ & $p$-Value & Significant & Regulate \\
\hline LDHA_MOUSE & 0.724713 & 1.004712 & 1.386359 & 0.471301 & 0.011192 & yes & up \\
\hline TRFE_MOUSE & 1.653381 & 0.731186 & 0.442237 & -1.17711 & 0.011198 & yes & down \\
\hline ODPA_MOUSE & 0.34843 & 1.081721 & 3.104561 & 1.634389 & 0.012074 & yes & up \\
\hline MURC_MOUSE & 0.539877 & 0.805455 & 1.491924 & 0.577174 & 0.016378 & yes & up \\
\hline TXNL1_MOUSE & 1.253474 & 0.942139 & 0.751623 & -0.41192 & 0.016452 & yes & down \\
\hline EPN3_MOUSE & 0.822867 & 1.572029 & 1.910429 & 0.933897 & 0.016926 & yes & up \\
\hline ANXĀ2_MOUSE & 0.78758 & 0.451204 & 0.572899 & -0.80365 & 0.01695 & yes & down \\
\hline MDHC_MOUSE & 0.737936 & 0.899583 & 1.219054 & 0.285762 & 0.017653 & yes & up \\
\hline SYNP2_MOUSE & 0.546814 & 1.127627 & 2.062178 & 1.044169 & 0.01788 & yes & up \\
\hline ATPO_MOUSE & 0.899583 & 2.080248 & 2.312457 & 1.209427 & 0.017984 & yes & up \\
\hline DEST_MOUSE & 1.349091 & 0.843344 & 0.62512 & -0.6778 & 0.018374 & yes & down \\
\hline VATB2_MOUSE & 1.616824 & 0.737951 & 0.45642 & -1.13156 & 0.018838 & yes & down \\
\hline MLRS_MOUSE & 0.644024 & 1.264857 & 1.963991 & 0.973788 & 0.02064 & yes & up \\
\hline AMPL_MOUSE & 1.507006 & 1.005905 & 0.667486 & -0.58319 & 0.021085 & yes & down \\
\hline GLYG_MOUSE & 0.676148 & 0.824278 & 1.21908 & 0.285793 & 0.023871 & yes & up \\
\hline AT2A1_MOUSE & 2.670282 & 7.878805 & 2.950552 & 1.560985 & 0.025035 & yes & up \\
\hline K1C27_MOUSE & 1.387947 & 0.415728 & 0.299527 & -1.73924 & 0.025627 & yes & down \\
\hline ITIH3_MOUSE & 1.978311 & 0.867782 & 0.438648 & -1.18887 & 0.028266 & yes & down \\
\hline RS11_MOUSE & 0.805592 & 0.505846 & 0.627919 & -0.67135 & 0.029542 & yes & down \\
\hline RM11_MOUSE & 0.883314 & 1.086471 & 1.229995 & 0.298652 & 0.029977 & yes & up \\
\hline RBMS2_MOUSE & 0.739031 & 1.081721 & 1.463701 & 0.549621 & 0.030714 & yes & up \\
\hline CYC_MOMOUSE & 0.594349 & 1.33729 & 2.250008 & 1.16993 & 0.031063 & yes & up \\
\hline K1C16__MOUSE & 1.231848 & 0.41048 & 0.333223 & -1.58544 & 0.031209 & yes & down \\
\hline K2C71_MOUSE & 1.331865 & 0.313541 & 0.235415 & -2.08672 & 0.032436 & yes & down \\
\hline K2C6A_MOUSE & 3.102133 & 0.347336 & 0.111967 & -3.15885 & 0.033321 & yes & down \\
\hline HSPB2_MOUSE & 0.563349 & 1.001528 & 1.777809 & 0.8301 & 0.033797 & yes & up \\
\hline NNTM_MOUSE & 0.973119 & 1.247436 & 1.281895 & 0.358278 & 0.033941 & yes & up \\
\hline COX41_MOUSE & 1.271059 & 1.889273 & 1.486377 & 0.5718 & 0.034524 & yes & up \\
\hline GCAB_MOUSE & 0.542972 & 0.317315 & 0.584403 & -0.77496 & 0.035724 & yes & down \\
\hline ALDOA_MOUSE & 0.217516 & 0.558516 & 2.567704 & 1.360479 & 0.03765 & yes & up \\
\hline PDLI5_MOUSE & 0.507804 & 1.323852 & 2.607016 & 1.382399 & 0.038285 & yes & up \\
\hline PGAM2_MOUSE & 0.544537 & 1.247436 & 2.290818 & 1.195863 & 0.038803 & yes & up \\
\hline NUCL_MOUSE & 1.214212 & 0.917008 & 0.755229 & -0.40501 & 0.038826 & yes & down \\
\hline TITIN_MOUSE & 0.525364 & 1.998309 & 3.803666 & 1.927391 & 0.0389 & yes & up \\
\hline NDUB4_MOUSE & 0.711696 & 0.887495 & 1.247013 & 0.318477 & 0.039585 & yes & up \\
\hline TM9S1_MOUSE & 0.661394 & 0.956005 & 1.44544 & 0.531508 & 0.040421 & yes & up \\
\hline LRC15_MOUSE & 0.769529 & 1.380911 & 1.794488 & 0.843572 & 0.042306 & yes & up \\
\hline GRP78_MOUSE & 1.316223 & 0.519738 & 0.39487 & -1.34055 & 0.044264 & yes & down \\
\hline MYOZ3_MOUSE & 0.498299 & 0.948807 & 1.90409 & 0.929101 & 0.0481 & yes & up \\
\hline EVPL_MOUSE & 1.009253 & 0.708314 & 0.70182 & -0.51083 & 0.048194 & yes & down \\
\hline ECP3_MOUSE & 0.732769 & 2.571728 & 3.509603 & 1.811308 & 0.048229 & yes & up \\
\hline KR151_MOUSE & 0.748812 & 0.476557 & 0.636418 & -0.65195 & 0.048479 & yes & down \\
\hline F16P2_MOUSE & 0.676664 & 1.004627 & 1.484676 & 0.570148 & 0.04869 & yes & up \\
\hline K2C73_MOUSE & 1.303273 & 0.655997 & 0.503346 & -0.99038 & 0.049896 & yes & down \\
\hline
\end{tabular}

The volcano diagram of differential proteins is shown in Figure 4A. GO enrichment analysis (Figure 4B) indicated that 13 functions were extremely significantly enriched $(p<0.001)$, for example, keratinization, carbohydrate catabolic and metabolic processes, intermediate filament cytoskeleton organization, hair follicle morphologenesis, lysosomal membrane, etc.; 27 functions were very significantly enriched $(p<0.01)$, for example, glucose metabolic process, hair cycle process, regulation of bone remodeling, mitochondrial electron transport cytochrome $\mathrm{c}$ to oxygen, vacuolar membrane, basal plasma membrane, misfolded protein binding, response to heat, etc.; others were significantly enriched $(p<0.05)$, such as positive regulation of apoptotic signaling pathway via death domain receptor, negative regulation of hydrogen peroxide metabolic process, regulation of interleukin-10 and -12 productions, etc.

KEGG (Kyoto Encyclopedia of Genes and Genomes) metabolic pathway analysis (Figure 4C) showed that only three pathways were significantly $(p<0.05)$ influenced: HIF-1 signaling pathway, glycolysis/gluconeogenesis, and methane metabolism. Other important pathways include RNA degradation, Alzheimer's disease, Leglonellosis, carbon metabolism, mineral absorption, insulin 
signaling pathway, collecting duct acid secretion, etc. Interaction network analysis by String demonstrated that 42 differential proteins were involved in the acting network of AAH (Figure 4D). Two core sub-networks existed: one was the sub-network centered by Pgam2, including Pygm, Mylpf, Atp2a1, Akdoa, Ldha, etc.; another was the sub-network that consisted of four proteins, i.e., Rps11, Rps3a, Rps21, and Mrp111. By iPath2.0 (http://pathways.embl.de/ipath2.cgi\#) analysis, the global view of the pathways involved in differential proteins is presented in Figure 4E. The major pathways include Oxidative phosphorylation, glutathione metabolism, Cysteine and methionine metabolism, amino sugars and nucleotides metabolism, etc. The differential proteins that mostly participated in these pathways include ODPA_MOUSE (K00161), F16P1_MOUSE (K03841), ENOA_MOUSE (K01689), MDHC_MOUSE (K00025), PGAM1_MOUSE (K01834).

In summary, proteomic analysis revealed that the top regulated proteins include Pygm (1.9 fold), Titin (1.9 fold), Ecp3 (1.8 fold), Hpt ( -3.4 fold), K2c7 ( -2.1 fold), and K2c6a ( -3.2 fold). The significantly enriched functions include $(p<0.001)$ keratinization, intermediate filament cytoskeleton organization, hair follicle morphologenesis and lysosomal membrane; but only three pathways were significantly $(p<0.05)$ regulated: HIF-1 signaling pathway, glycolysis/ gluconeogenesis, and methane metabolism. The network analysis of differential proteins demonstrated the existence of two core sub-networks: one was the sub-network centered by Pgam2; another was the sub-network that consisted of four proteins (Rps11, Rps3a, Rps21, and Mrpl11).

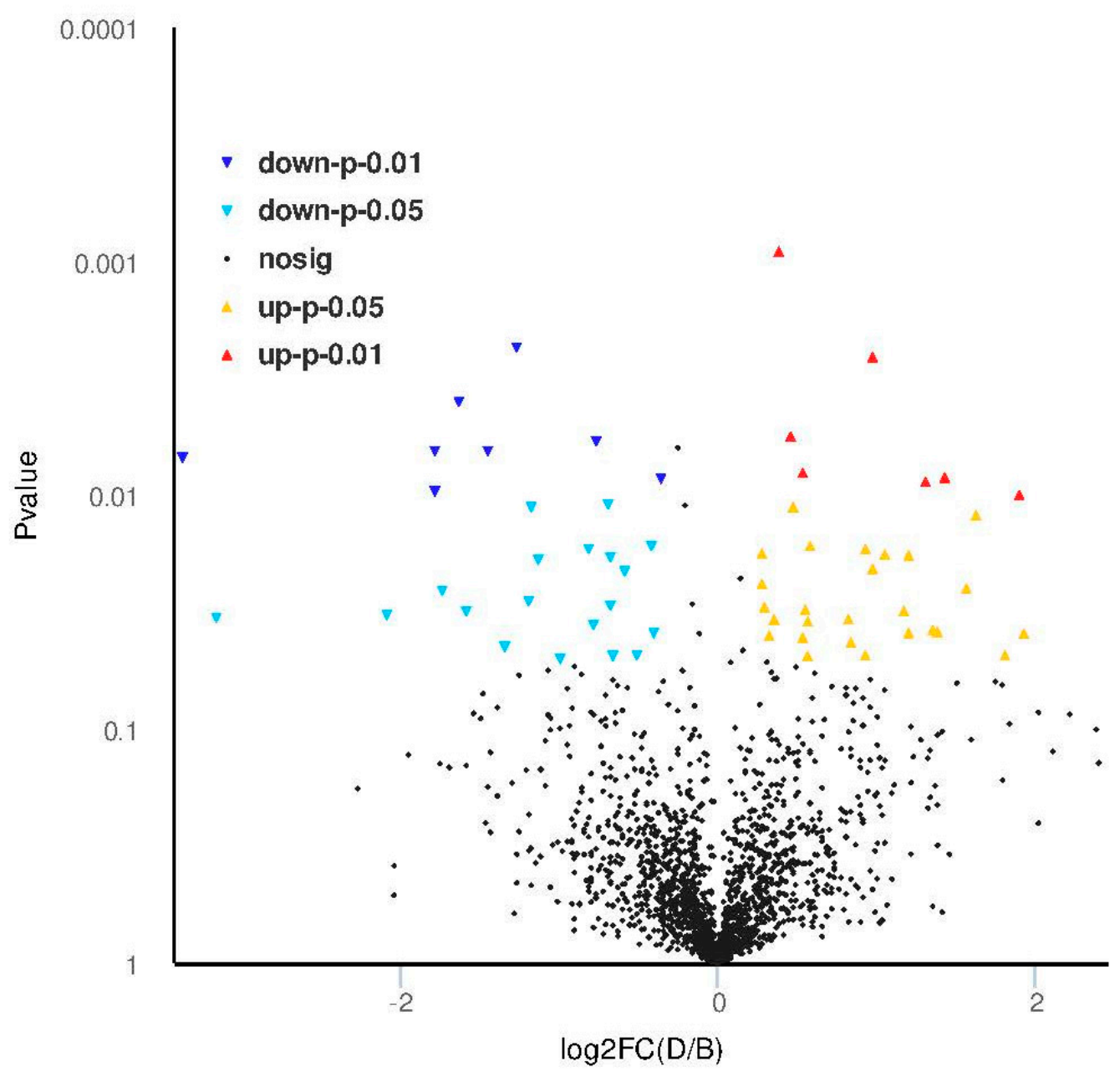

(A)

Figure 4. Cont. 


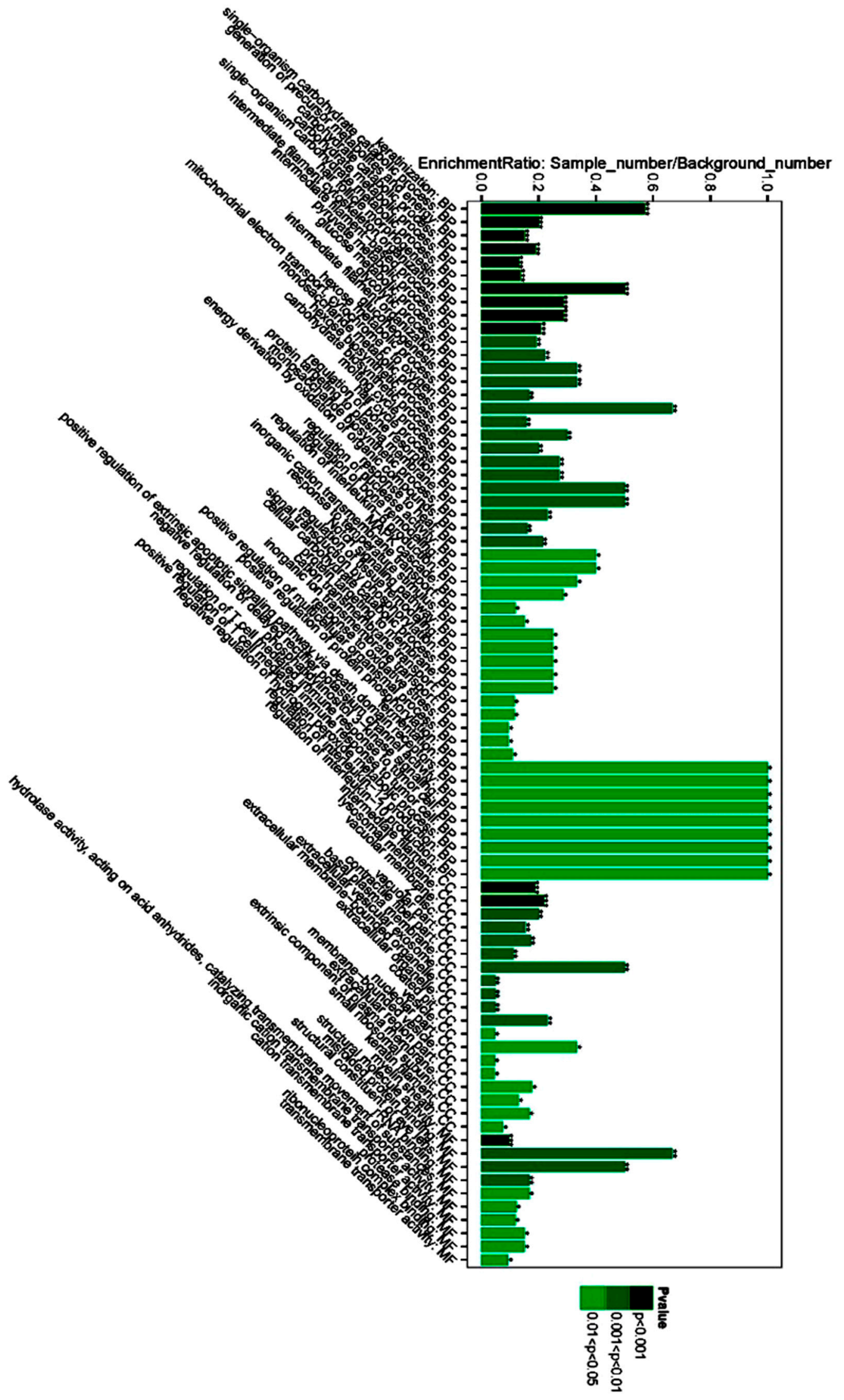

(B)

Figure 4. Cont. 


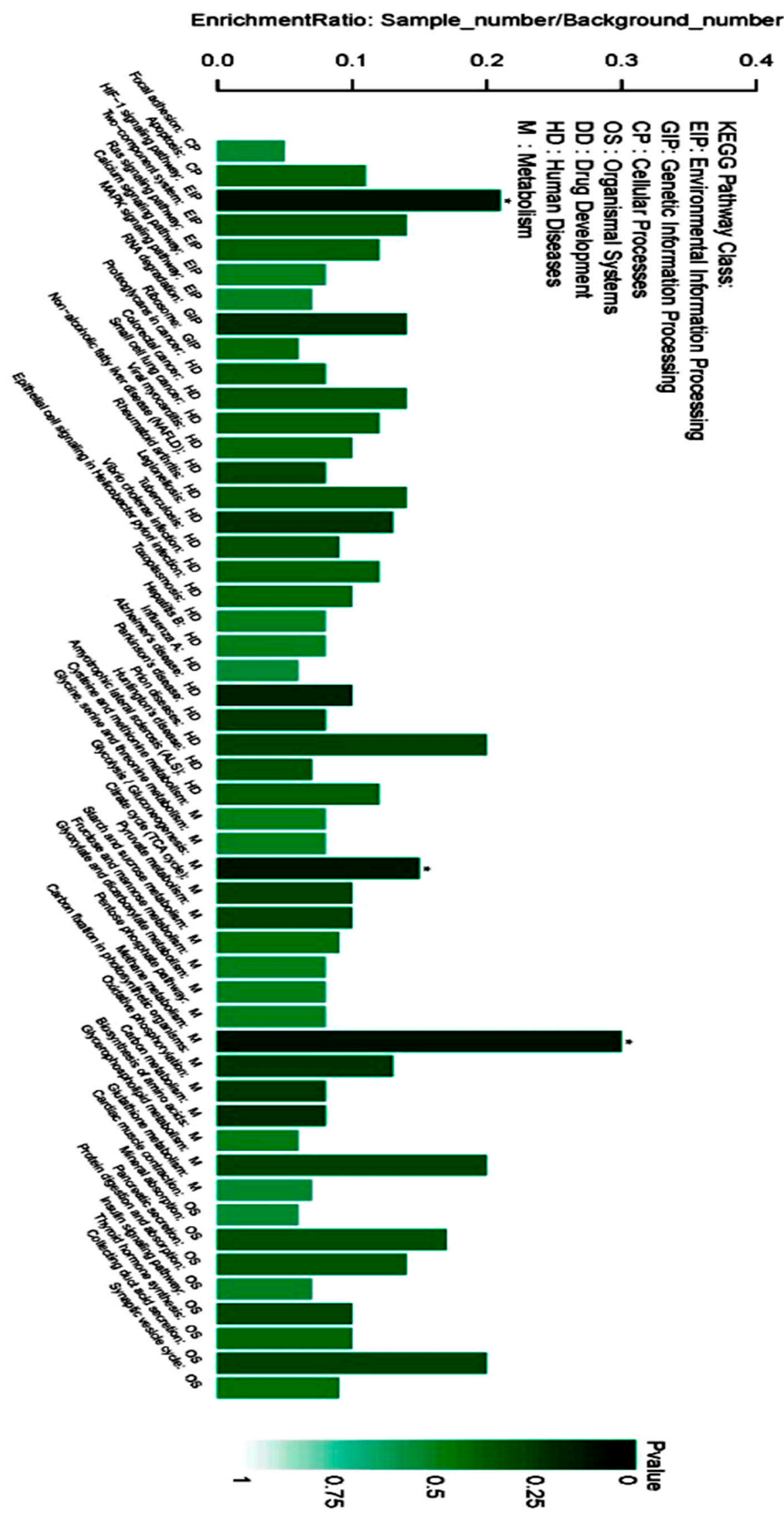

(C)

Figure 4. Cont. 


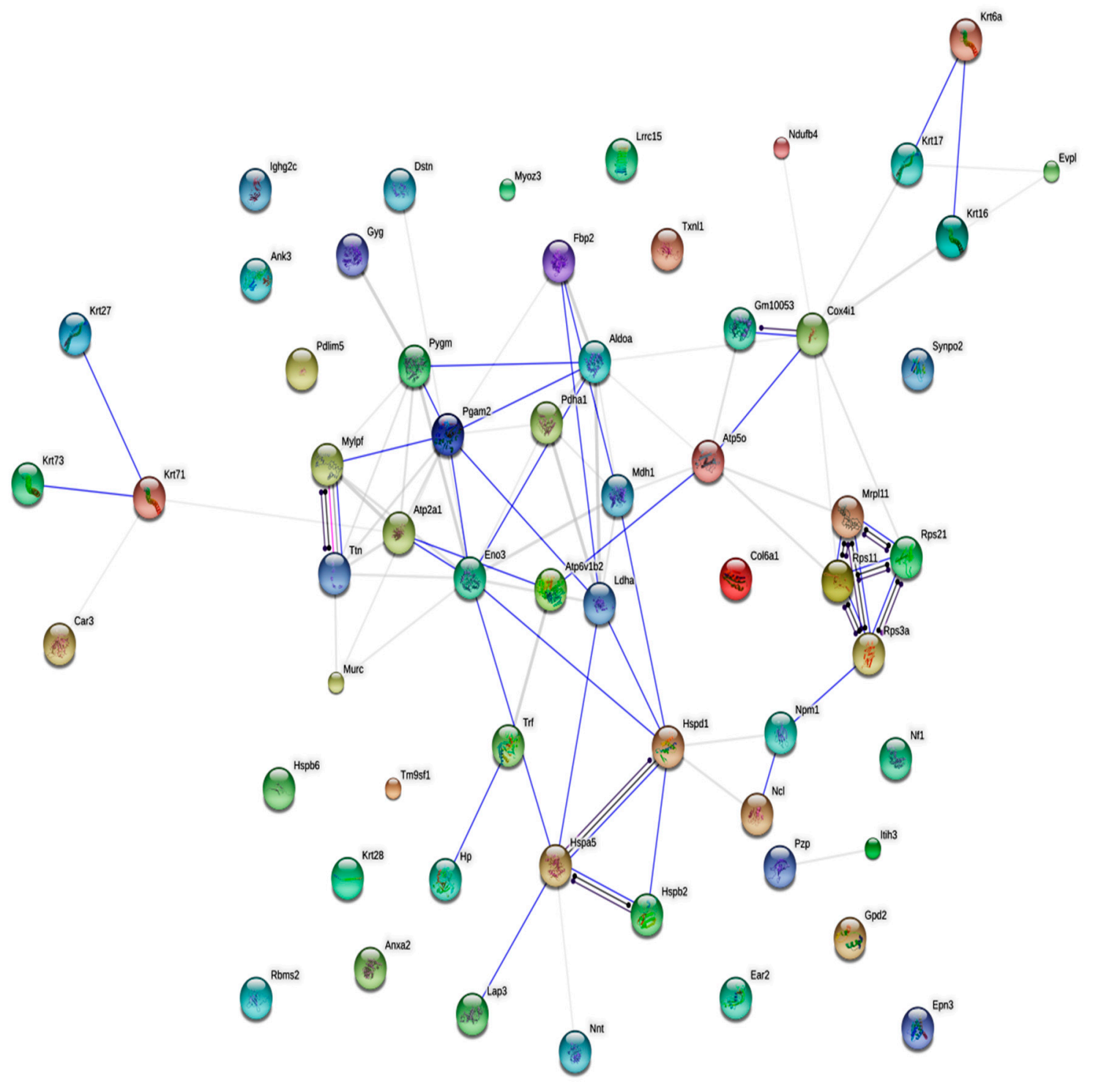

(D)

Figure 4. Cont. 


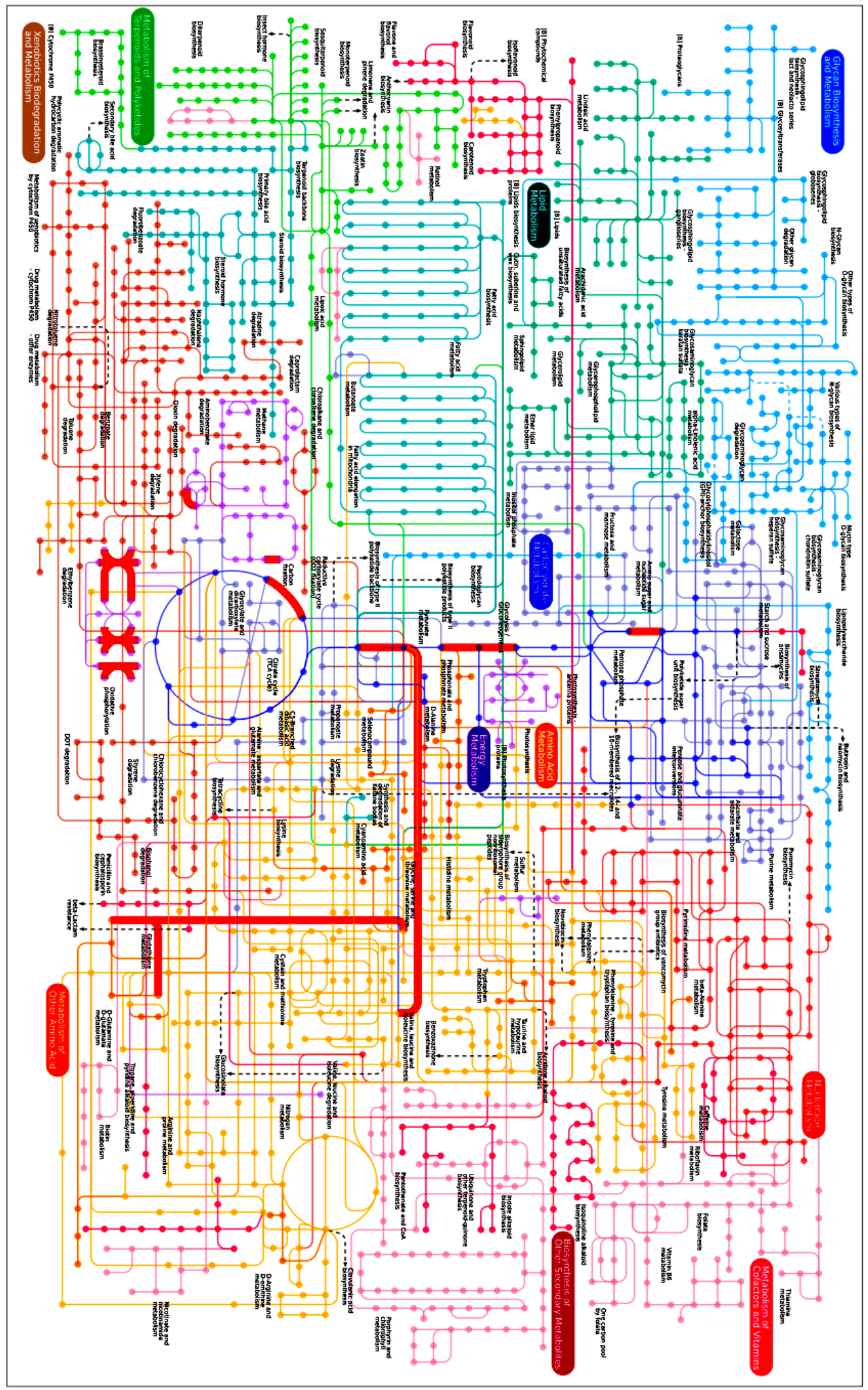

(E)

Figure 4. Volcano diagram (A), GO enrichment analysis. 1 keratinization: BP; 2 single-organism carbohydrate catabolic process: $\mathrm{BP} ; 3$ generation of precursor metabolites and energy: $\mathrm{BP} ; 4$ carbohydrate catabolic process: BP; 5 carbohydrate metabolic process: BP; 6 single-organism carbohydrate metabolic 
process: BP; 7 hair follicle morphogenesis: BP; 8 intermediate filament cytoskeleton organization: BP; 9 intermediate filament-based process: BP; 10 pyruvate metabolic process: BP; 11 glucose metabolic process: BP; 12 glycolytic process: BP; 13 intermediate filament organization: BP; 14 gluconeogenesis: $\mathrm{BP} ; 15$ hexose metabolic process: $\mathrm{BP} ; 16$ mitochondrial electron transport, cytochrome c to oxygen: $\mathrm{BP} ; 17$ monosaccharide metabolic process: $\mathrm{BP} ; 18$ hexose biosynthetic process: $\mathrm{BP} ; 19$ carbohydrate biosynthetic process: BP; 20 molting cycle process: BP; 21 hair cycle process: BP; 22 regulation of bone resorption: BP; 23 protein targeting to plasma membrane: BP; 24 monosaccharide biosynthetic process: BP; 25 energy derivation by oxidation of organic compounds: BP; 26 response to heat: BP; 27 regulation of nuclease activity: BP; 28 regulation of bone remodeling: BP; 29 regulation of interleukin- 6 production: $\mathrm{BP} ; 30$ MAPK cascade: $\mathrm{BP} ; 31$ inorganic cation transmembrane transport: $\mathrm{BP} ; 32$ response to temperature stimulus: BP; 33 Notch signaling pathway: BP; 34 regulation of tissue remodeling: BP; 35 signal transduction by phosphorylation: BP; 36 cellular carbohydrate catabolic process: BP; 37 protein targeting to membrane: BP; 38 cation transmembrane transport: BP; 39 inorganic ion transmembrane transport: $\mathrm{BP} ; 40$ response to oxidative stress: $\mathrm{BP} ; 41$ positive regulation of multicellular organismal process: BP; 42 positive regulation of protein phosphorylation: BP; 43 fermentation: $\mathrm{BP} ; 44$ positive regulation of extrinsic apoptotic signaling pathway via death domain receptors: BP; 45 negative regulation of delayed rectifier potassium channel activity: BP; 46 phosphatidylinositol 3-kinase signaling: BP; 47 regulation of $\mathrm{T}$ cell mediated immune response to tumor cell: BP; 48 positive regulation of $\mathrm{T}$ cell mediated immune response to tumor cell: $\mathrm{BP} ; 49$ negative regulation of hydrogen peroxide metabolic process: BP; 50 regulation of interleukin-12 production: BP; 51 regulation of interleukin-10 production: BP; 52 intermediate filament: CC; 53 lysosomal membrane: CC; 54 vacuolar membrane: CC; $55 \mathrm{Z}$ disc: CC; 56 vacuolar part: CC; 57 contractile fiber part: CC; 58 basal plasma membrane: CC; 59 extracellular vesicular exosome: CC; 60 extracellular membrane-bounded organelle: CC; 61 extracellular organelle: CC; 62 coated pit: CC; 63 vesicle: CC; 64 nucleolar part: CC; 65 membrane-bounded vesicle: CC; 66 extracellular region part: CC; 67 extrinsic component of plasma membrane: CC; 68 small ribosomal subunit: CC; 69 keratin filament: CC; 70 myelin sheath: CC; 71 structural molecule activity: MF; 72 misfolded protein binding: MF; 73 structural constituent of eye lens: MF; 74 rRNA binding: MF; 75 hydrolase activity, acting on acid anhydrides, catalyzing transmembrane movement of substances: MF; 76 inorganic cation transmembrane transporter activity: MF; 77 cation transmembrane transporter activity: MF; 78 protease binding: MF; 79 ribonucleoprotein complex binding: MF; 80 transmembrane transporter activity: MF (B). KEGG metabolic pathways. 1 Focal adhesion: CP; 2 Apoptosis: CP; 3 HIF-1 signaling pathway: EIP; 4 Two-component system: EIP; 5 Ras signaling pathway: EIP; 6 Calcium signaling pathway: EIP; 7 MAPK signaling pathway: EIP; 8 RNA degradation: GIP; 9 Ribosome: GIP; 10 Proteoglycans in cancer: HD; 11 Colorectal cancer: HD; 12 Small cell lung cancer: HD; 13 Viral myocarditis: HD; 14 Non-alcoholic fatty liver disease (NAFLD): HD; 15 Rheumatoid arthritis: HD; 16 Legionellosis: HD; 17 Tuberculosis: HD; 18 Vibrio cholerae infection: HD; 19 Epithelial cell signaling in Helicobacter pylori infection: HD; 20 Toxoplasmosis: HD; 21 Hepatitis B: HD; 22 Influenza A: HD; 23 Alzheimer's disease: HD; 24 Parkinson's disease: HD; 25 Prion diseases: HD; 26 Huntington's disease: HD; 27 Amyotrophic lateral sclerosis (ALS): HD; 28 Cysteine and methionine metabolism: M; 29 Glycine, serine and threonine metabolism: M; 30 Glycolysis/Gluconeogenesis: M; 31 Citrate cycle (TCA cycle): M; 32 Pyruvate metabolism: M; 33 Starch and sucrose metabolism: M; 34 Fructose and mannose metabolism: M; 35 Glyoxylate and dicarboxylate metabolism: M; 36 Pentose phosphate pathway: M; 37 Methane metabolism: M; 38 Carbon fixation in photosynthetic organisms: M; 39 Oxidative phosphorylation: M; 40 Carbon metabolism: M; 41 Biosynthesis of amino acids: M; 42 Glycerophospholipid metabolism: M; 43 Glutathione metabolism: M; 44 Cardiac muscle contraction: OS; 45 Mineral absorption: OS; 46 Pancreatic secretion: OS; 47 Protein digestion and absorption: OS; 48 Insulin signaling pathway: OS; 49 Thyroid hormone synthesis: OS; 50 Collecting duct acid secretion: OS; 51 Synaptic vesicle cycle: OS (C). Interaction network identified by STRING (D) and by IPATH (E) for differential proteins related to the protection effect of the acetylated and amidated hexapeptide. 


\subsection{Western Blot Verification}

As mentioned above, proteomics identified 60 differential proteins between the UVB irradiation group and AAH treatment group. From them, six proteins were selected for verification by Western blot: Haptoglobin, Cytochrome c, Nucleophosmin, HSP60, CA3, PDHA1, and two parallels per sample. Expression intensities were determined by the digital gel image analysis system (LG2000, Hangzhou LongGene Scientific Instrument Co., Ltd. Hangzhou, China). Results revealed that all six proteins displayed consistent alterations between ITRAQ and Western blot experiments (Figure 5 and Table 2).

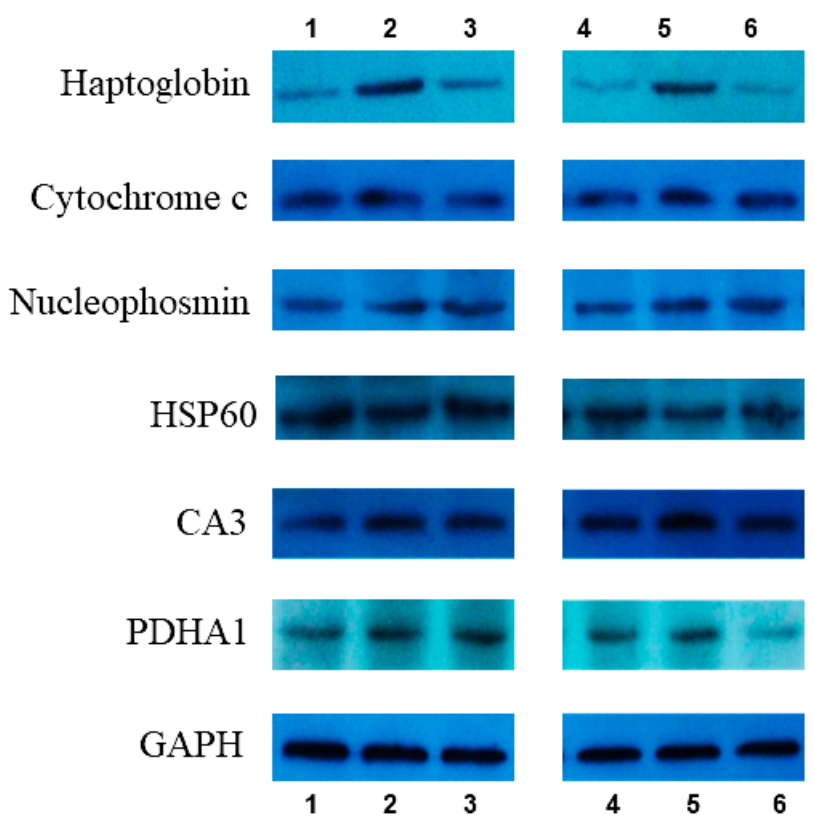

Figure 5. Validation of partly differentially expressed protein by Western blot: 1 and 4 come from the normal group; 2 and 5 come from the model group; 3 and 6 come from the acetylated and amidated hexapeptide group(Ac-GMCCSR-NH2).

Table 2. Validation of differential proteins from iTRAQ by Western blot (WB).

\begin{tabular}{ccc}
\hline \multirow{2}{*}{ Protein Name } & \multicolumn{2}{c}{ D/B } \\
\cline { 2 - 3 } & iTRAQ & WB \\
\hline Haptoglobin & -3.36 & -2.33 \\
Cytochrome c & 1.17 & 1.23 \\
Nucleophosmin & -0.68 & -1.22 \\
HSP60 & -1.45 & -1.16 \\
CA3 & 1.31 & 0.75 \\
PDHA1 & 1.63 & 1.04 \\
\hline
\end{tabular}

\section{Discussion}

Except for intrinsic aging, photoaging is a major process of skin aging. Keratinocytes are the outermost layer of the skin, constituting $95 \%$ of the cells in the epidermis. UV radiation induces apoptosis of keratinocytes to form sunburn cells, showing premature and abnormal keratinization [14]. Tsoyi et al. [15] proved that anthocyanins from black soybean seed coats protected Hacats from UVB-induced apoptosis. Lee et al. [16] reported that the processed Panax ginseng, Sun Ginseng, has an anti-apoptotic effect on UVB-irradiated Hacats. The present study indicated that AAH had much lower toxicity on Hacats than the positive control matrixyl ( $81.52 \%$ vs. $5.32 \%)$. Additionally, AAH possessed significantly stronger ability $(p<0.05)$ to proliferate UVB-damaged Hacats than the positive 
control matrixyl (137.14\% vs. $121.56 \%$ ). This suggests better potential of AAH as a skin care agent in terms of toxicity and protective effect on keratinocytes, compared to the positive control matrixyl.

Following keratinocytes experiment, the protective effect of AAH on mice skin was tested. The specific characteristics of photoaging included epidermal thickening and inflammatory infiltration [17]. Indeed, Feng et al. [18] reported that UV irradiation increased, by over 2-fold, the epidermal thickness of mice, while patchouli alcohol, the major active sesquiterpene found in Pogostemonis Herba, decreased by over $20 \%$ of the epidermal thickness. Meanwhile, UV exposure led to severe wrinkling with deep furrows, laxity and erythema in the skin of mice, but patchouli alcohol inhibited the formation of UV-induced erythema, roughness, and deep wrinkles. Similarly, Wu et al. [19] found that UVB resulted in an over 3-fold increase in the skin thickness compared with that of normal mice, and induced erythema and inflammation of the mice skin, Coffea arabica extract decreased the epidermal thickness by $30 \%$, and ameliorated UVB-induced inflammation and erythema. Our data also indicated that the thickness of mice skin after UVB irradiation $(40.2 \mu \mathrm{m})$ was dramatically increased, over 3-fold compared to the thickness of normal mice skin $(13.1 \mu \mathrm{m})$, while AAH reversed the increased thickness of mice skin $(13.5 \mu \mathrm{m})$. On the other hand, UVB-irradiated mice skin displayed vacuolar degeneration of epidermal basal cells and inflammatory cells infiltration of the dermis, while AAH removed the vacuolar degeneration and inflammatory cells infiltration.

It is well known that UV irradiation induces oxidative damage by inhibiting the activities of endogenous antioxidant enzymes such as SOD and GSH-Px, elevating the production of MDA (a well-known biomarker for lipid peroxidation) [20]. In addition, UV irradiation alters the connective tissues of the skin by up-regulating the expression of MMPs, which play an important role in skin photoaging. For example, by cleaving type I and type III collagen, MMP- 1 initiates collagen breakdown; after being activated by MMP-3, MMP-1, -2 , and -9 derived from dermal fibroblasts or keratinocytes initiate degradation of type I and III collagens [21]; MMP-2 and MMP-9 are important mediators in UV-irradiated skin damage and in the formation of wrinkles [22]. Lee et al. [23] showed that by suppressing the NF-kappaB pathway, cordycepin down-regulated MMP-1 and -3 gene expression in UVB-irradiated human dermalfibroblasts. Feng et al. [18] indicated that patchouli alcohol markedly reversed the decreased activities of SOD and GSH-Px, reduced MDA production by $30 \%$, and inhibited the increase of MMP-1 and MMP-3 expression by about $24.6 \%$ and $39.3 \%$ respectively, in UVB-treated mice. Kim et al. [24] revealed that youngiaside increased the expression of SOD and suppressed MMP-1 production via Nrf2 and AMPK pathways in Hacats and Human Dermal Fibroblasts. Wu et al. [25] reported that Coffea arabica extract attenuated UVB-induced MMP-1 expression in the hairless mouse skin. The present study demonstrated that compared with the UVB-irradiated mice group, AAH reduced MDA content by 49\%; increased SOD, CAT, and GSH-Px activities by $103 \%, 49 \%$, and $116 \%$, respectively; decreased MMP- 1 and MMP-3 expressions by $27 \%$ and $29 \%$, respectively; which exhibited better effects than the positive control matrixyl.

Although too many studies of UVB damage were reported, the molecular mechanisms causing this damage requires further elucidation. Over the past few years, proteomic tools have been used to investigate the biological effects of UVB exposure on human skin. For example, Bertrand-Vallery et al. [26] performed a 2D-DIGE proteomic profiling of human keratinocytes undergoing UVB-induced alternative differentiation, 69 differentially abundant proteins were identified by mass spectrometry, especially, confirming TRIpartite Motif Protein 29 as a survival factor. Wu et al. [25] employed lysine- and cysteine-labeling 2D-DIGE and MALDI-TOF mass spectrometry to conduct proteomic analysis of UVB-induced protein expression in skin fibroblasts. The results showed that 89 significantly changed proteins were identified, these UVB-modulated proteins were involved in many cellular responses including photoaging, melanogenesis, anti-apoptosis, tumorigenesis, and cell migration. Fang et al. [17] applied a high-throughput 2DE analysis coupled with MALDI-TOF MS to profile the global proteins involved in chronologically aged and photoaged skin in nude mice, and 15 differential proteins were identified. The most striking characteristic was that 14-3-3 sigma was 
down-regulated by 3.41-fold in the chronological aging skin, and proliferating cell nuclear antigen was up-regulated by 1.5-fold in UVB-induced aging skin.

In the present study, a novel iTRAQ-based proteomics tool was applied to obtain new insights into the protein profiles involved in photoaged mice skin. Compared with UVB treatment group, 60 differential proteins were identified in AAH treatment group. These proteins formed a complex acting network consisting of two core sub-networks and were involved in three major pathways: HIF-1 signaling pathway, glycolysis/gluconeogenesis, and methane metabolism.

Among the significantly up-regulated proteins, the proteins with a little link to aging include ODPA (Pyruvate dehydrogenase E1 component subunit alpha) (1.6 fold) and AT2A1 (Sarcoplasmic/endoplasmic reticulum calcium ATPase 1, SERCA1) (1.6 fold). It was reported that pyruvate dehydrogenase was decreased in senescent skin fibroblasts [27]; increased expression of SERCA can reduce hydroxyl radical injury in murine myocardium [28]. The most remarkedly down-regulated protein was HPT (Haptoglobin), which was 3.4-fold reduced in the modified hexapeptide group compared to the UVB group. Haptoglobin is an acute-phase protein secreted by white adipose tissue or liver cells. HPT is induced in pro-oxidative conditions such as systemic inflammation or obesity. In both inflammation and obesity HPT is up-regulated [29]. K2C6A (Keratin 6A) was also a remarkedly altered protein with 3.2-fold reduction. Similarly, other keratins were also decreased, including K1C17 (keratin 17) ( -1.8 fold), K1C28 (keratin 28) ( -1.8 fold), K1C27 (keratin 27) ( -1.7 fold), K1C16 (keratin 16) ( -1.6 fold), and K2C71 (keratin 71$)$ ( -2.1 fold). It was reported that keratin-6 was significantly higher $(p<0.05)$ in elderly skin [30]. The aging of skin is associated with decreased barrier function and gradual deterioration of the epidermal immune response, leading to chronic inflammation. Depianto et al. [31] pointed out that absence of keratin 17 attenuated hyperplasia and inflammation in models of acute dermatitis. High expression of keratin 16 in mice skin could cause skin lesions and alterations in keratin filament organization and in cell adhesion [32]. Thus, the above-mentioned disappearance of inflammatory cells infiltration could be related to attenuation of skin inflammation caused by significant down-regulation of HPT, K2C6A, and K1C17.

In conclusion, this is the first study to demonstrate the anti-photoaging effects of an acetylated and amidated peptide in UVB-irradiated keratinocytes and mice. The results showed that AAH reduced the content of MDA, increased the activities of SOD, CAT, and GSH-Px, and decreased the expression of MMP-1 and MMP-3. By employing a novel iTRAQ-based proteomic analysis, 60 differential proteins were identified, which were mapped into an interaction network composed of two core sub-networks, and key pathways were determined. In a word, the present data suggests that AAH was superior to the positive control matrixyl and has strong potential to be developed into a cosmetic product against skin photoaging.

Author Contributions: Data curation, J.W.; Investigation, Q.Z. (Qiaohui Zeng); Validation, Q.Z. (Qiuchan Zhou); Writing—original draft, X.Z.; Writing-review and editing, J.J.

Funding: This study was supported by Ocean and Fisheries Development Project (A201401C08) from Administration of Ocean and Fisheries of Guangdong Province.

Conflicts of Interest: The authors declare no conflict of interest.

\section{References}

1. Na, C.R.; Wang, S.; Kirsner, R.S.; Federman, D.G. Elderly adults and skin disorders: Common problems for nondermatologists. South Med. J. 2012, 105, 600-606. [CrossRef] [PubMed]

2. Kong, S.Z.; Shi, X.G.; Feng, X.X.; Li, W.J.; Liu, W.H.; Chen, Z.W.; Xie, J.H.; Lai, X.P.; Zhang, S.X.; Zhang, X.J.; et al. Inhibitory effect of hydroxysafflor yellow a on mouse skin photoaging induced by ultraviolet irradiation. Rejuvenation Res. 2013, 16, 404-413. [CrossRef] [PubMed]

3. Brenneisen, P.; Sies, H.; Scharffetter-Kochanek, K. Ultraviolet-B irradiation and matrix metalloproteinases: From induction via signaling to initial events. Ann. N. Y. Acad. Sci. 2002, 973, 31-43. [CrossRef] [PubMed] 
4. Debacq-Chainiaux, F.; Borlon, C.; De Hertogh, B.; Remacle, J.; Morvan, P.Y.; Vallée, R.; Toussaint, O. Identification of potential anti-photoageing algal compounds using an in-vitro model of photoageing. J. Pharm Pharmacol. 2006, 58, 1577-1583. [CrossRef] [PubMed]

5. Neyrinck, A.M.; Taminiau, B.; Walgrave, H.; Daube, G.; Cani, P.D.; Bindels, L.B.; Delzenne, N.M. Spirulina Protects against Hepatic Inflammation in Aging: An Effect Related to the Modulation of the Gut Microbiota? Nutrients 2017, 9, 633. [CrossRef] [PubMed]

6. Sadek, K.M.; Lebda, M.A.; Nasr, S.M.; Shoukry, M. Spirulina platensis prevents hyperglycemia in rats by modulating gluconeogenesis and apoptosis via modification of oxidative stress and MAPK-pathways. Biomed. Pharmacother. 2017, 92, 1085-1094. [CrossRef] [PubMed]

7. Wu, Q.; Liu, L.; Miron, A.; Klímová, B.; Wan, D.; Kuca, K. The antioxidant, immunomodulatory, and antiinflammatory activities of Spirulina: An overview. Arch. Toxicol. 2016, 90, 1817-1840. [CrossRef] [PubMed]

8. Souza, C.; Campos, P.M.; Schanzer, S.; Albrecht, S.; Lohan, S.B.; Lademann, J.; Darvin, M.E.; Meinke, M.C. Radical scavenging activity of a sunscreen enriched by antioxidants providing protection in the whole solar spectral range. Eur. J. Pharm Sci. 2017. [CrossRef]

9. Zeng, Q.H.; Fan, X.D.; Zheng, Q.P.; Wang, J.J.; Zhang, X.W. Anti-oxidant, hemolysis inhibition, and collagen-stimulating activities of a new hexapeptide derived from Arthrospira (Spirulina) platensis. J. Appl. Phycol. 2018, 30, 1655-1665. [CrossRef]

10. Wallace, R.J. Acetylation of peptides inhibits their degradation by rumen micro-organisms. Br. J. Nutr. 1992, 68, 365-372. [CrossRef]

11. Kim, K.H.; Seong, B.L. Peptide Amidation: Production of Peptide Hormones in vivo and in vitro. Biotechnol. Bioprocess. Eng. 2001, 6, 244-251. [CrossRef]

12. Thomas, A. Towards a Functional Understanding of Protein N-Terminal Acetylation. PLoS Biol. 2011, 9, e1001074.

13. Song, K.H.; Kim, S.B.; Shim, C.K.; Chung, S.J.; Kim, D.D.; Rhee, S.K.; Choi, G.J.; Kim, C.H.; Kim, K. Paracellular permeation-enhancing effect of AT1002 C-terminal amidation in nasal delivery. Drug Des. Devel Ther. 2015, 9 , 1815-1823. [CrossRef] [PubMed]

14. Sheehan, J.M. Young AR the sunburn cell revisited: An update on mechanistic aspects. Photochem. Photobiol. Sci. 2002, 1, 365-377. [CrossRef] [PubMed]

15. Tsoyi, K.; Park, H.B.; Kim, Y.M.; Chung, J.I.; Shin, S.C.; Shim, H.J.; Lee, W.S.; Seo, H.G.; Lee, J.H.; Chang, K.C.; et al. Protective effect of anthocyanins from black soybean seed coats on UVB-induced apoptotic cell death in vitro and in vivo. J. Agric. Food Chem. 2008, 56, 10600-10605. [CrossRef] [PubMed]

16. Lee, H.; Lee, J.Y.; Song, K.C.; Kim, J.; Park, J.H.; Chun, K.H.; Hwang, G.S. Protective Effect of Processed Panax ginseng, Sun Ginseng on UVB-irradiated Human Skin Keratinocyte and Human Dermal Fibroblast. J. Ginseng Res. 2012, 36, 68-77. [CrossRef] [PubMed]

17. Fang, J.Y.; Wang, P.W.; Huang, C.H.; Chen, M.H.; Wu, Y.R.; Pan, T.L. Skin aging caused by intrinsic or extrinsic processes characterized with functional proteomics. Proteomics 2016, 16, 2718-2731. [CrossRef] [PubMed]

18. Feng, X.X.; Yu, X.T.; Li, W.J.; Kong, S.Z.; Liu, Y.H.; Zhang, X.; Xian, Y.F.; Zhang, X.J.; Su, Z.R.; Lin, Z.X. Effects of topical application of patchouli alcohol on the UV-induced skin photoaging in mice. Eur. J. Pharm Sci. 2014, 63, 113-123. [CrossRef]

19. Wu, P.Y.; Huang, C.C.; Chu, Y.; Huang, Y.H.; Lin, P.; Liu, Y.H.; Wen, K.C.; Lin, C.Y.; Hsu, M.C.; Chiang, H.M. Alleviation of Ultraviolet B-Induced Photodamage by Coffea arabica Extract in Human Skin Fibroblasts and Hairless Mouse Skin. Int. J. Mol. Sci. 2017, 18, 782. [CrossRef] [PubMed]

20. Pillai, S.; Oresajo, C.; Hayward, J. Ultraviolet radiation and skin aging: Roles of reactive oxygen species, inflammation and protease activation, and strategies for prevention of inflammation-induced matrix degradation-A review. Int. J. Cosmet. Sci. 2005, 27, 17-34. [CrossRef]

21. Nagase, H.; Visse, R.; Murphy, G. Structure and function of matrix metalloproteinases and TIMPs. Cardiovasc. Res. 2006, 69, 562-573. [CrossRef] [PubMed]

22. Steinbrenner, H.; Ramos, M.C.; Stuhlmann, D.; Sies, H.; Brenneisen, P. UVA-mediated downregulation of MMP-2 and MMP-9 in human epidermal keratinocytes. Biochem. Biophys. Res. Commun. 2003, 308, 486-491. [CrossRef] 
23. Lee, Y.R.; Noh, E.M.; Jeong, E.Y.; Yun, S.K.; Jeong, Y.J.; Kim, J.H.; Kwon, K.B.; Kim, B.S.; Lee, S.H.; Park, C.S.; et al. Cordycepin inhibits UVB-induced matrix metalloproteinase expression by suppressing the NF-kappaB pathway in human dermal fibroblasts. Exp. Mol. Med. 2009, 41, 548-554. [CrossRef] [PubMed]

24. Kim, M.; Park, Y.G.; Lee, H.J.; Lim, S.J.; Nho, C.W. Youngiasides A and C Isolated from Youngia denticulatum Inhibit UVB-Induced MMP Expression and Promote Type I Procollagen Production via Repression of MAPK/AP-1/NF-kB and Activation of AMPK/Nrf2 in HaCaT Cells and Human Dermal Fibroblasts. J. Agric. Food Chem. 2015, 63, 5428-5438. [CrossRef]

25. Wu, C.L.; Chou, H.C.; Cheng, C.S.; Li, J.M.; Lin, S.T.; Chen, Y.W.; Chan, H.L. Proteomic analysis of UVB-induced protein expression-and redox-dependent changes in skin fibroblasts using lysine- and cysteine-labeling two-dimensional difference gel electrophoresis. J. Proteom. 2012, 75, 1991-2014. [CrossRef]

26. Bertrand-Vallery, V.; Belot, N.; Dieu, M.; Delaive, E.; Ninane, N.; Demazy, C.; Raes, M.; Salmon, M.; Poumay, Y.; Debacq-Chainiaux, F.; et al. Proteomic profiling of human keratinocytes undergoing UVB-induced alternative differentiation reveals TRIpartite Motif Protein 29 as a survival factor. PLoS ONE 2010, 5, e10462. [CrossRef]

27. Wei, Y.H.; Wu, S.B.; Ma, Y.S.; Lee, H.C. Respiratory function decline and DNA mutation in mitochondria, oxidative stress and altered gene expression during aging. Chang Gung Med. J. 2009, 32, 113-132.

28. Hiranandani, N.; Bupha-Intr, T.; Janssen, P.M. SERCA overexpression reduces hydroxyl radical injury in murine myocardium. Am. J. Physiol Heart Circ. Physiol. 2006, 291, H3130-H3135. [CrossRef]

29. Lisi, S.; Gamucci, O.; Vottari, T.; Scabia, G.; Funicello, M.; Marchi, M.; Galli, G.; Arisi, I.; Brandi, R.; D'Onofrio, M.; et al. Obesity-associated hepatosteatosis and impairment of glucose homeostasis are attenuated by haptoglobin deficiency. Diabetes 2011, 60, 2496-2505. [CrossRef]

30. Kinn, P.M.; Holdren, G.O.; Westermeyer, B.A.; Abuissa, M.; Fischer, C.L.; Fairley, J.A.; Brogden, K.A.; Brogden, N.K. Age-dependent variation in cytokines, chemokines, and biologic analytes rinsed from the surface of healthy human skin. Sci. Rep. 2015, 5, 10472. [CrossRef]

31. Depianto, D.; Kerns, M.L.; Dlugosz, A.A.; Coulombe, P.A. Keratin 17 promotes epithelial proliferation and tumor growth by polarizing the immune response in skin. Nat. Genet. 2010, 42, 910-914. [CrossRef] [PubMed]

32. Wawersik, M.J.; Mazzalupo, S.; Nguyen, D.; Coulombe, P.A. Increased levels of keratin 16 alter epithelialization potential of mouse skin keratinocytes in vivo and ex vivo. Mol. Biol. Cell. 2001, 12, 3439-3450. [CrossRef] [PubMed] 